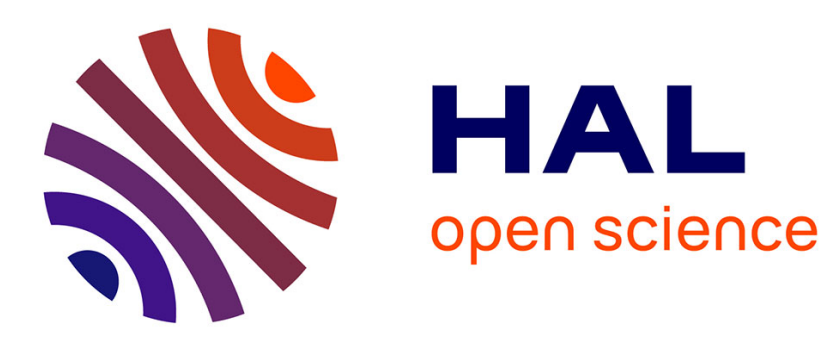

\title{
Dynamics of nonlinear dissipative systems in the vicinity of resonance
}

\author{
K.Y. Plaksiy, Yuri Mikhlin
}

\section{To cite this version:}

K.Y. Plaksiy, Yuri Mikhlin. Dynamics of nonlinear dissipative systems in the vicinity of resonance. Journal of Sound and Vibration, 2015, 334, pp.319-337. 10.1016/j.jsv.2014.09.001 . hal-01350800

\section{HAL Id: hal-01350800 \\ https://hal.science/hal-01350800}

Submitted on 1 Aug 2016

HAL is a multi-disciplinary open access archive for the deposit and dissemination of scientific research documents, whether they are published or not. The documents may come from teaching and research institutions in France or abroad, or from public or private research centers.
L'archive ouverte pluridisciplinaire HAL, est destinée au dépôt et à la diffusion de documents scientifiques de niveau recherche, publiés ou non, émanant des établissements d'enseignement et de recherche français ou étrangers, des laboratoires publics ou privés. 


\title{
Dynamics of nonlinear dissipative systems in the vicinity of resonance
}

\author{
K.Y. Plaksiy, Y.V. Mikhlin \\ Department of Applied Mathematics, National Technical University “KhPI”, Frunze str. 21, Kharkov 61002, Ukraine
}

\begin{abstract}
The behavior of nonlinear dissipative 2-DOF mechanical systems in the vicinity of resonance is studied in this paper. Namely, the free resonance vibrations of a spring-mass-pendulum system and the forced resonance vibrations of a 2-DOF dissipative system containing a nonlinear absorber are considered. A reduced system stated with respect to the system energy, the arctangent of the vibration amplitudes ratio, and the phase difference, is obtained and analyzed. The nonlinear normal mode approach is used in this analysis. Conditions for vibration energy localization are discussed.
\end{abstract}

\section{Introduction}

An investigation of the behavior of nonlinear systems in the presence of internal resonance is an important step in the solution of some theoretical and applied problems, in particular, problems of modal interaction and the localization of vibration energy. Internal resonance can lead to a loss of vibration mode stability, and to the appearance of new vibration regimes as a result of bifurcation. In addition, the problem of primary external resonance is very important for nonlinear systems.

We refer to some important publications of the last decades on the resonance problems. Different results for internal and external resonance problems and numerous applications are presented in the book [1] by Nayfeh and Mook and in the book [2] by Avramov and Mikhlin [2]. The book [3] by Manevitch and Manevich is devoted to internal resonances in different mechanical systems, including autonomous and non-autonomous two-DOF nonlinear systems, infinite chains of particles, circular rings and cylindrical shells. Internal resonances are investigated by using an approach based on introducing a complex representation of the equations of motion together with the multiple scales method. Steady-state and non-stationary regimes of motion, an interaction of internal and forced resonances, and bifurcations of steady-state modes and their stability are all studied.

The transfer of energy caused by internal resonance is discussed in various publications. A description of the energy transfer was presented in the work of Witt and Gorelik [4]. The authors considered a spring pendulum for which they showed that the transfer of angular oscillation energy to vertical oscillation energy, and back, takes place for a natural frequency ratio of 2:1. For some initial conditions this transfer is absent, and partial subsystems are synchronized. The transfer of energy caused by internal resonance was also investigated by Struble and Heinbockel [5,6], Tsel'man [7], Merceret al. [8]. Energy transients in Lyapunov systems, and in systems close to these, was analyzed in the book by Starzhinskii [9]. The dynamics of a spring pendulum, a pendulum with elastic suspension, and elementary particles in cyclic accelerators were all considered. The determination of periodic solutions and regions of instability in the system parameter space are described by parametric resonance theory. Forking periodic solutions which appear under this resonance condition are also investigated. Then, asymptotic methods are used to describe the energy transfer. The 
energy transient from one nonlinear vibration mode to another is considered by Nayfeh and Mook [1]. In [10] it was shown that for some conditions this transfer from high-frequency modes to low-frequency mode is possible, and is caused by modal interaction, and the one-way channeling of energy is not obligatory. In a series of papers by Vakakis, Manevitch, Gendelman, Bergman et al. [11-14], a theoretical investigation and an experimental verification of the energy transfer and localization has been presented for different nonlinear systems. The authors of these works describe a process of spacial energy transfer from the location of their initial appearance to a nonlinear absorber, where the energy is localized.

Nonlinear energy interactions can occur due to internal resonances, and energy transfers may be realized due to nonlinear mode bifurcations. Some principal results in this area are summarized in the book by Vakakis et al. [15]. In the book by Morozov [16] the influence of internal and external resonances on the global dynamics of autonomous and nonautonomous systems is investigated. Problems of limit circles and regular and non-regular behavior are considered.

In publications [2,3,11-15] nonlinear normal modes (NNMs) are used, and the NNMs are considered to be an important element of investigation. The Kauderer-Rosenberg concept of NNMs, first proposed in [17-19], is based on the determination of trajectories in the nonlinear system configuration space. In this concept some generalized coordinate is chosen as the independent coordinate instead of time, and other generalized coordinates are presented as univalent functions of the chosen coordinate. Numerous publications describe the problem of the existence of NNMs, methods for construction of the regimes, an analysis of the stability of NNMs, a generalization of the concept of NNMs to non-autonomous systems, and to systems with non-smooth characteristics etc. The NNM theory is applied to the analysis of the dynamics of some applied mechanical systems. Some principal results of the NNM theory and its applications are presented in [2,20-22].

Accounting for dissipation introduces additional complications in the analysis of nonlinear system dynamics in the vicinity of resonance. In particular, the interaction of vibration modes, their stability, the localization of energy and other important characteristics of dynamical process as all depend on time, since dissipative system energy decreases in time. In addition to this, vibration modes in dissipative systems are not the classical Kauderer-Rosenberg vibration modes because modes in dissipative systems contain an exponential decrease in the vibration amplitude. Therefore, it can be observed that so-called transient nonlinear normal modes (TNNMs) exist only for some specific value of the system energy, and they disappear when the energy decreases. In spite of the instability of these transient modes other motions of the dissipative system can still approach the transient modes when the system energy is close to some specific energy value. We suppose that the use of so-called reduced systems as was used earlier for non-dissipative systems [23,24], permits the description of principal elements of nonlinear dissipative system behavior. The reduced system is written with respect to the system total energy, the arctangent of the ratio of amplitudes and the difference of phases. Numerical simulation confirms some principal results obtained from analytical investigation.

In the paper two nonlinear dissipative elastic systems are considered. Namely, the resonant behavior of a spring-masspendulum is considered in Section 2. Then an investigation of a 2-DOF system containing a small mass nonlinear absorber under external periodic excitation is presented in Section 3. In this Section external resonances involving the first and the second fundamental frequencies are analyzed. In addition, a case of combined external and internal resonances is also considered. An analysis of all resonances is made by the use of reduced systems. Simultaneously an investigation of the stability and bifurcation of vibration modes is performed. The evolution of such vibration modes, and the energy transfer from one mode to another one, are both considered. The transient nonlinear normal modes are selected and their influence on the system behavior is investigated. The localization of energy is discussed and analytical results are compared with numerical and numerical-analytical simulations.

\section{Resonance behavior of a spring-mass-pendulum system}

A spring-mass-pendulum system is shown in Fig. 1. Introducing the following parameters and independent variable, $\omega_{1}=\sqrt{k /(M+m)}, \quad \omega_{2}=\sqrt{g / l}, \quad p=\omega_{1} / \omega_{2}, \quad \alpha=m /(m+M), \tau=\omega_{2} t$ one presents equations of motion of the system as

$$
\left\{\begin{array}{l}
\ddot{x}+p^{2} x+2 \varepsilon \eta_{x} \dot{x}-\alpha\left(\ddot{\theta} \sin \theta+\dot{\theta}^{2} \cos \theta\right)=0 \\
\ddot{\theta}+2 \varepsilon \eta_{\theta} \dot{\theta}+\sin \theta-\ddot{x} \sin \theta=0
\end{array}\right.
$$

There are two nonlinear normal modes in the system (1) without dissipation: the localized $x$-mode of vertical vibrations $(x=x$ $(t), \theta=0)$, and the non-localized pendulum mode $(x=x(t), \theta=\theta(t))$ when both vibration amplitudes are of the same order.

A procedure of transformation to so-called reduced system is used to analyze the behavior of the system (1) in the vicinity of the main parametric resonance. At the first step, the detuning parameter $\Delta$ is introduced into the first equation of the system (1) by means of the next relation: $p^{2}-4+4 \varepsilon^{2} \eta_{\theta}^{2}-\varepsilon^{2} \eta_{x}^{2}=\varepsilon \Delta$.

Introducing the following scaling of coordinates, $x \rightarrow \varepsilon X, \theta \rightarrow \varepsilon \theta$, one applies to Eq. (1) the classical multiple scales method [25]. Namely, one introduces new independent variables as $T_{n}=\varepsilon^{n} t(n=0,1,2, \ldots)$. A solution is represented by the following asymptotic series: $x=x_{0}\left(T_{0}, T_{1}, \ldots\right)+\varepsilon x_{1}\left(T_{0}, T_{1}, \ldots\right)+\ldots, \theta=\theta_{0}\left(T_{0}, T_{1}, \ldots\right)+\varepsilon \theta_{1}\left(T_{0}, T_{1}, \ldots\right)$. It leads to the following systems of equations for the first and second approximations by the different orders of the small parameter $\varepsilon$ :

$$
\left\{\begin{array}{l}
\frac{\partial^{2} x_{0}}{\partial T_{0}^{2}}+4 x_{0}=0 \\
\frac{\partial^{2} \theta_{0}}{\partial T_{0}^{2}}+\theta_{0}=0
\end{array}\right.
$$




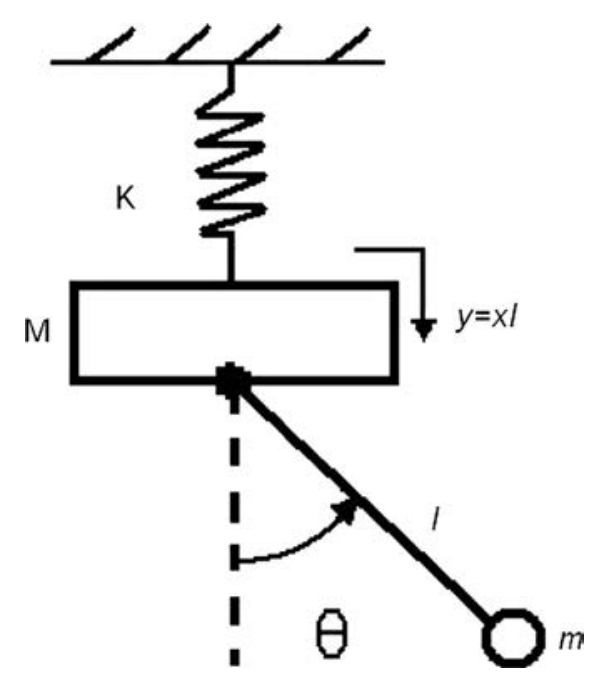

Fig. 1. Spring-mass-pendulum system.

$$
\left\{\begin{array}{l}
\frac{\partial^{2} x_{1}}{\partial T_{0}^{2}}+4 x_{1}=-2 \frac{\partial^{2} x_{0}}{\partial T_{1} \partial T_{0}}+\alpha\left(\frac{\partial^{2} \theta_{0}}{\partial T_{0}^{2}}\right) \theta_{0}+\alpha\left(\frac{\partial \theta_{0}}{\partial T_{0}}\right)^{2}-2 \eta_{x} \frac{\partial x_{0}}{\partial T_{0}}-x_{0} \Delta \\
\frac{\partial^{2} \theta_{1}}{\partial T_{0}^{2}}+\theta_{1}=-2 \frac{\partial^{2} \theta_{0}}{\partial T_{1} \partial T_{0}}+\left(\frac{\partial^{2} x_{0}}{\partial T_{0}^{2}}\right) \theta_{0}-2 \eta_{\theta} \frac{\partial \theta_{0}}{\partial T_{0}}
\end{array}\right.
$$

The system (2) solution is presented in the form

$$
\left\{\begin{array}{c}
x_{0}=A_{x}\left(T_{1}\right) e^{2 i T_{0}}+\bar{A}_{x}\left(T_{1}\right) e^{-2 i T_{0}} \\
\theta_{0}=A_{\theta}\left(T_{1}\right) e^{i T_{0}}+\bar{A}_{\theta}\left(T_{1}\right) e^{-i T_{0}} .
\end{array}\right.
$$

The solution (4) is substituted into the system of the second approximation (3), and secular generating terms are eliminated. The following change of variables, $A_{x}=a_{x} e^{i \beta_{x}}, A_{\theta}=a_{\theta} e^{i \beta_{\theta}}$, leads to the following equations written with respect to amplitudes and phases of the solution (4)

$$
\left\{\begin{array}{l}
a_{x}^{\prime}=\frac{\alpha}{2} a_{\theta}^{2} \sin \varphi-\eta_{x} a_{x} \\
a_{\theta}^{\prime}=-2 a_{x} a_{\theta} \sin \varphi-\eta_{\theta} a_{\theta} \\
\beta_{x}^{\prime}=\frac{\alpha}{2} \frac{a_{\theta}^{2}}{a_{x}} \cos \varphi+\frac{\Delta}{4} \\
\beta_{\theta}^{\prime}=2 a_{x} \cos \varphi
\end{array}\right.
$$

where $\varphi=\beta_{x}-2 \beta_{\theta}$.

The reduced system stated with respect to the system (5) energy $K$, the arctangent of the ratio of amplitudes $\psi$ and the difference of phases $\varphi$, can be obtained by means of the following change of variables: $a_{x}=K \sin \psi, a_{y}=K$ cos $\psi$. After some transformations the reduced system are presented in the following form:

$$
\left\{\begin{array}{c}
K^{\prime}=-K\left(\eta_{x} \cos ^{2} \psi+\eta_{\theta} \sin ^{2} \psi\right) \\
\psi^{\prime}=K \sin \psi\left(-\sqrt{\alpha} \sin \varphi+\left(\eta_{x}-\eta_{\theta}\right) \cos \psi\right) \\
\varphi^{\prime}=\left(\sqrt{\alpha} K \frac{\sin ^{2} \psi}{\cos \psi}-2 \sqrt{\alpha} K \cos \psi\right) \cos \varphi+\frac{\Delta}{4}
\end{array}\right.
$$

Then equilibrium positions of the system (6) as functions of the parameters $\Delta, \alpha$ and the variable $K$ are analyzed. Using the second equation of the system (6) one can find the following equilibrium positions: $\sin \psi=0$ (it corresponds to the vertical vibration mode), or $\sin \varphi=\left(\left(\eta_{x}-\eta_{\theta}\right) / \sqrt{\alpha}\right) \cos \psi$ (it corresponds to the coupled vibration mode).

One can obtain from the third equation of the system (6) that $\cos \psi=(\Delta / 4) \pm \sqrt{\left(\Delta^{2} / 16\right)+3 \alpha K^{2} \cos ^{2} \varphi / 3 K} \cos \varphi \sqrt{\alpha}$. According to the expression at the right hand side, $\psi$ is the function of $K(\psi=\psi(K))$, thus condition $\psi^{\prime}=0$ cannot be realized. So, motions of the system, corresponding to this equilibrium, are not realized in the form of the classical nonlinear normal modes by Kauderer-Rosenberg; they are equivalent to the classical NNMs only if the dissipation is absent.

If $\eta_{x} \equiv \eta_{\theta}$ one has $\sin \varphi=0$. In this case the first equilibrium point, which is denoted as $P_{1}$, is determined from the third equation of the system (6) by the next relations: $\sin \varphi=0$ and $\cos \psi=(\Delta / 4)+\sqrt{\left(\Delta^{2} / 16\right)+3 \alpha K^{2}} / 3 K \sqrt{\alpha}$. A condition of existence of $\cos \psi$ gives us the inequality $\Delta / 4 \sqrt{\alpha} K \leq 1$; therefore $0 \leq \psi \leq \pi / 2$. Other equilibrium point, which is denoted as $P_{2}$, is determined from the third Eq. (17) by the following relations: $\sin \varphi=0$ and $\cos \psi=(\Delta / 4)+\sqrt{\left(\Delta^{2} / 16\right)+3 \alpha K^{2}} / 3 K \sqrt{\alpha}$. 
It exists in the following region of the system parameters: $-1 \leq \Delta / 4 \sqrt{\alpha} K$. Note that if $-1 \leq \Delta / 4 \sqrt{\alpha} K \leq 1$ then both equilibrium points exist.

In Figs. 2,3 the dependence $\varphi(\psi)$ is presented for the case when the system is situated in a region of the bifurcation existence (Fig. 2), and for the case when the system is not in this region (Fig. 3), respectively. The straight line $\psi=0$ corresponds to localized vibrations. To simulate the first case we solve Eq. (6) numerically by the Runge-Kutta method, choosing initial values from the following region: $0 \leq \psi(0) \leq \pi / 2,0 \leq \varphi(0) \leq \pi$, for $K(0)=0.9, \Delta=0.12, \alpha=0.1, \eta_{x}=\eta_{\theta}=0.01$.

In Fig. 2 one can see a simultaneous existence of few equilibrium positions of the reduced system (6) corresponding to the region of bifurcation. In the vicinity of resonance there are two modes of coupled vibrations together with a single mode of localized vibrations. The modes of coupled vibrations correspond to described above equilibrium positions $P_{1}$ and $P_{2}$. These positions move in time along straight lines $\varphi=0$ and $\varphi=\pi$. Namely these straight lines correspond to coupled vibrations of the system (1). Besides, it is clear that for $\eta_{x} \equiv \eta_{\theta}$ the energy $K$ decreases in time. So, for some fixed value of the detuning parameter $\Delta$ we obtain the following result: for small values of the initial energy the system under consideration is in the region where only a single equilibrium position exists, that is, the bifurcation does not takes place; otherwise, for not small values of the initial energy the system can be situated in region where two modes of the coupled vibration exist, but in the course of time the system will get into a region with a single equilibrium position. Namely, when $|\Delta / 4 \sqrt{\alpha} K|>1$, the bifurcation disappears. In Fig. 2 the transition from the lower equilibrium position to the upper one corresponds to the time instant when the lower equilibrium position ceases to exist, but the upper one continues to exist, that is to the moment of the bifurcation disappearance. So, depending on the energy level of the system it can obtain a region where vertical vibrations lose stability as a result of bifurcation. Then, when the energy decreases, the system leaves this region; the bifurcation disappears, and the vertical vibration mode becomes stable again.

An analysis of trajectories presented in Fig. 2 shows that trajectories remain near the equilibrium positions $P_{1}$ and $P_{2}$, realizing spiral-like motions near straight lines $\varphi=0$ and $\varphi=\pi$, and do not remain near the straight line $\psi=0$, which corresponds to vertical vibrations. Thus, the coupled vibrations of the system are stable in the region where the bifurcation exists, and the vertical mode loses stability.

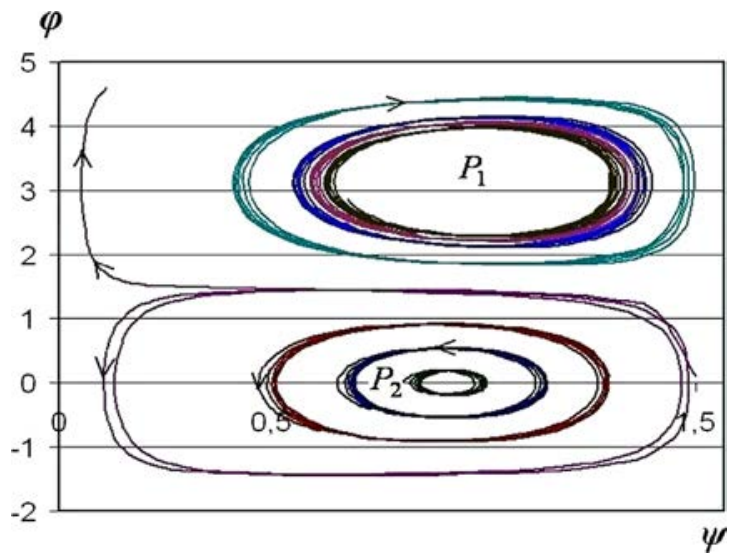

Fig. 2. Dependence $\varphi(\psi)$. Bifurcation of vertical vibrations exist.

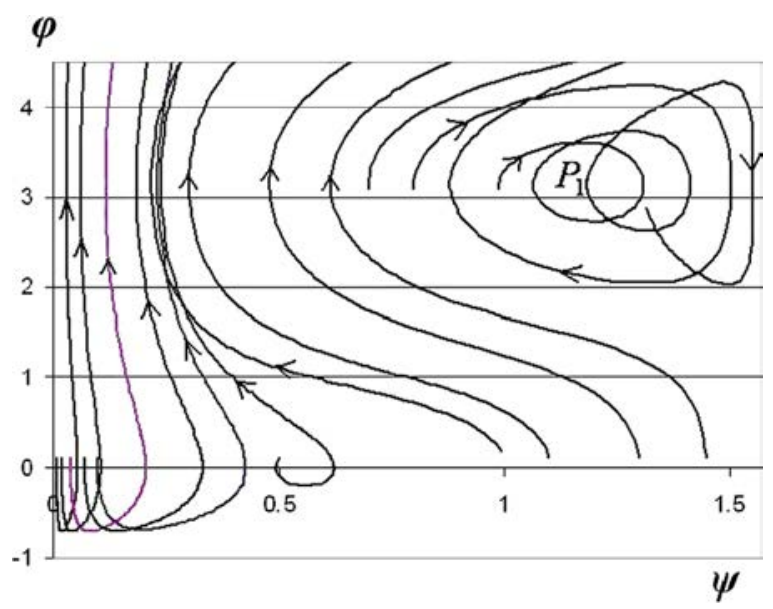

Fig. 3. Dependence $\varphi(\psi)$. Bifurcation of vertical vibrations does not exist. 
A case when only a single equilibrium position exists is illustrated in Fig. 3. Trajectories were obtained numerically by the Runge-Kutta method from Eq. (6); initial values were chosen from the region $0 \leq \psi(0) \leq \pi / 2,0 \leq \varphi(0) \leq \pi$, for $K(0)=0.37$, $\Delta=0.5, \alpha=0.1, \eta_{x}=\eta_{\theta}=0.01$. Fig. 3 shows that the trajectories approach in time the straight line $\psi=0$, which corresponds to vertical vibrations of the system (1) and withdraw from the straight line $\varphi=\pi$, that is, from the equilibrium position $P_{1}$, by spiral-like trajectories. That is, in the region where bifurcation does not occur, the coupled vibration mode is unstable, and the vertical vibration mode is stable.

A behavior of the initial system (1) in the region where bifurcation exists is illustrated in Fig. 4. Trajectories in the system configuration space were obtained numerically for initial values $\theta(0)=0.001, x(0)=0.09, \dot{x}(0)=0.03, \dot{\theta}(0)=0.0002$ and for $\alpha=0.1, p=2, \eta_{x}=\eta_{\theta}=0.01$. One can see in Fig. 4 a the development of vertical vibrations instability and the transient to the regime of two modes of coupled vibrations interaction. Coupled vibrations are shown in Fig. 4b for larger values of time than in Fig. $4 \mathrm{a}$.

For the region where the bifurcation does not exist and the vertical vibration mode is stable near resonance, the system (5) is solved numerically on the interval $\tau \in[0,150]$ for initial values $a_{x}(0)=0.02, a_{\theta}(0)=0.002, \beta_{x}(0)=0.01, \beta_{\theta}(0)=0.01$, and for $\alpha=0.1, \eta_{x}=\eta_{\theta}=0.01, \Delta=0.05$. The obtained numerical solution is introduced to the first approximation solution of the system (1) in the vicinity of resonance (see relations (4)), which can be written as $x=2 a_{x}$ cos $\left(\beta_{x}+2 \tau\right), \theta=2 a_{\theta} \cos \left(\beta_{\theta}+\tau\right)$. Namely dependences of $x$ and $\theta$ on time, and trajectories in the place $x, \theta$, are presented in Figs. 5,6. Note that corresponding initial conditions for the variables $x$ and $\theta$ are the following: $\theta(0)=0.00039998$, $x(0)=0.039998, \dot{x}(0)=-0.00160495, \dot{\theta}(0)=-1.19995 \mathrm{e}-005$. Direct numerical integration of Eq. (1) by the Runge-Kutta method on the interval $\tau \in[0,150]$ for initial conditions presented above confirms a reliability of the results showed in Figs. 5,6.

To analyze a stability of the vertical vibrations of the system (1) in the vicinity of resonance, an analysis of the vertical vibration stability must be carried out on the basis of the linearized equations in variations, where variations $u$, $v$ are introduced by means of the relations $x=\tilde{x}+u, \theta=\tilde{\theta}+v$ :

$$
\left\{\begin{array}{l}
\ddot{u}+p^{2} u+2 \varepsilon \eta_{x} \dot{u}-\alpha(\ddot{\tilde{\theta}} v+2 \dot{\tilde{\theta}} \dot{v}+\ddot{v} \tilde{\theta})=0 \\
\ddot{v}+v+2 \varepsilon \eta_{\theta} \dot{v}-\tilde{\tilde{x}} \cdot v-\ddot{u} \tilde{\theta}=0
\end{array}\right.
$$

(a)

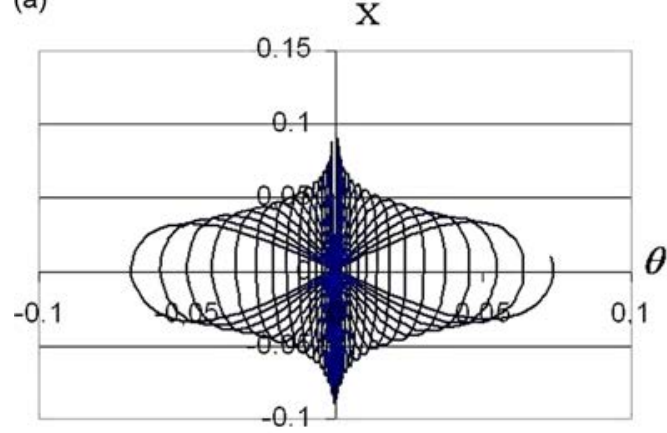

(b)

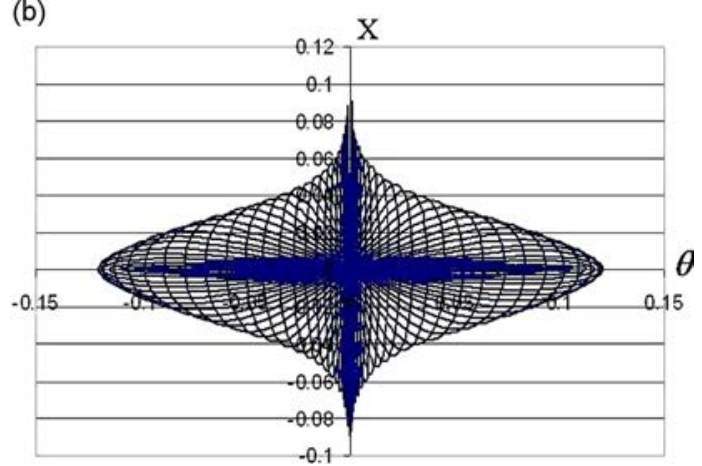

Fig. 4. Dependence $x(\theta)$ in region where bifurcation exists. (a) Dependence $x(\theta)$ for $\tau \in[0,100]$; (b). dependence $x(\theta)$ for $\tau \in[0,500]$.

(a) $\mathrm{X}$

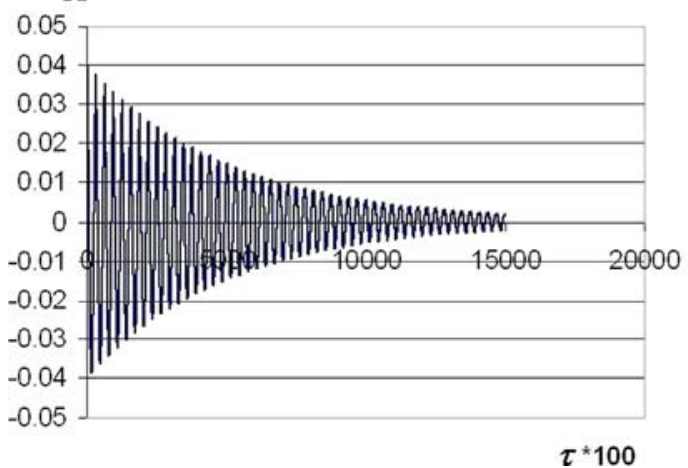

(b)

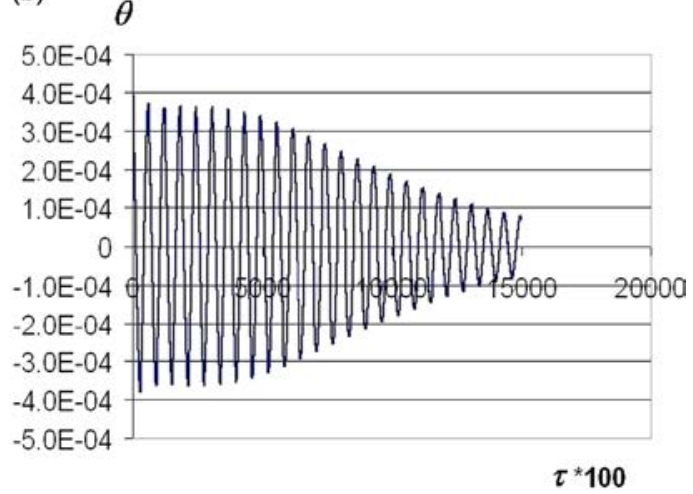

Fig. 5. Solution in time in the region where bifurcation does not exists. (a) Dependence $x(t)$; (b) dependence $\theta(t)$. 


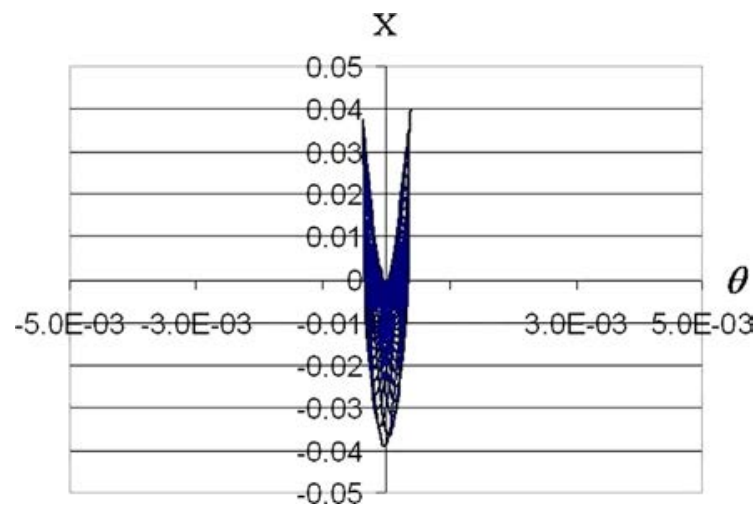

Fig. 6. Trajectory in the configuration place $x, \theta$ in the region where bifurcation does not exists and the vertical vibration mode is stable.

Stability of the vertical vibration mode is determined by angle variations. Thus, only the second equation of the system (7) is considered. The vertical mode vibration can be presented as

$$
\tilde{\theta}(\tau) \equiv 0, \tilde{x}(\tau)=A e^{-\varepsilon \eta_{x} \tau} \cos \omega_{x} \tau,
$$

where $\omega_{x}=\sqrt{p^{2}-\varepsilon^{2} \eta_{x}^{2}}$.

Substituting the relation (8) to the second Eq. (7), one has the following equation in variations:

$$
\ddot{v}+v+2 \varepsilon \eta_{\theta} \dot{v}-v\left(-\omega_{x}^{2} A e^{-\varepsilon \eta_{x} \tau} \cos \omega_{\chi} \tau+2 \varepsilon \eta_{x} \omega_{x} A e^{-\varepsilon \eta_{\chi} \tau} \sin \omega_{x} \tau+\varepsilon^{2} \eta_{x}^{2} A e^{-\varepsilon \eta_{x} \tau} \cos \omega_{x} \tau\right)=0
$$

Introducing the time scales $T_{0}=\tau, T_{1}=\varepsilon \tau$, and new variable $r=\omega_{x} T_{0} / 2$ and designations $a=4 / \omega_{x}^{2}, \mu=4 A e^{-\eta_{x} T_{1}}$, $v^{\prime}=\mathrm{d} v / \mathrm{d} r, \quad v^{\prime \prime}=\mathrm{d}^{2} v / \mathrm{d} r^{2}$, one can obtain the following equations in partial derivatives:

$$
\begin{gathered}
\varepsilon^{0}: v_{0}^{\prime \prime}+v_{0}(a+\mu \cos 2 r)=0 \\
\varepsilon^{1}: v_{1}^{\prime \prime}+v_{1}(a+\mu \cos 2 r)=-\frac{4 \eta_{\theta}}{\omega_{x}} v_{0}^{\prime}-\frac{4}{\omega_{x}} \frac{\partial v_{0}^{\prime}}{\partial T_{1}}+\frac{\eta_{x}}{2 \omega_{x}} \mu v_{0} \sin 2 r
\end{gathered}
$$

Eq. (10) is the Mathieu equation. Boundaries of stability/instability regions for this equation are well-known $[1,26]$. In particular, boundaries for the main parametric resonance can be written as

$$
\left\{\begin{array}{l}
a=1-\frac{1}{2} \mu-\frac{1}{32} \mu^{2}+\frac{1}{512} \mu^{3}-\frac{1}{24576} \mu^{4}+\ldots \\
a=1+\frac{1}{2} \mu-\frac{1}{32} \mu^{2}-\frac{1}{512} \mu^{3}-\frac{1}{24576} \mu^{4}+\ldots
\end{array} .\right.
$$

Additional analysis shows that the Eq. (11) does not essentially affect boundaries (12). It is obvious that boundaries (12) depend on time since $\mu=4 A e^{-\eta_{x} \varepsilon \tau}$. Boundaries (12) have been constructed for $\varepsilon=0.1, \eta_{x}=0.2$ and for the following instants of time: $\tau=0, \tau=50$ and $\tau=100$ (Fig. 7). Here the instability is observed inside of the boundary lines. Region of instability is narrowed over time, that is, vertical vibrations which are unstable at the beginning of the process become eventually stable. This result corresponds to one obtained by analysis of the reduced system: when the system energy decreases, then the bifurcation disappears and vertical vibrations become stable.

\section{Resonance behavior of nonlinear dissipative system under the external periodic excitation}

Presented above approach can be applied for analysis of the resonance dynamics of dissipative system which contains a nonlinear absorber, under external periodic excitation (Fig. 8).

\subsection{Investigation of external resonance on the first fundamental frequency}

One considers the following equations of motion:

$$
\left\{\begin{array}{l}
M \ddot{x}+\beta \dot{x}+c x+c_{2}(x-y)+\gamma(x-y)^{3}=r \sin \Omega t \\
m \ddot{y}+\beta \dot{y}-c_{2}(x-y)-\gamma(x-y)^{3}=0
\end{array}\right.
$$

where $M$ is the mass of the main system, $c=c_{0}+c_{1}, m$ is the mass of the nonlinear sub-system. It is introduced a small parameter to the system (13) assuming that the system is under the external periodic excitation $F(t)=r \sin \Omega t$; nonlinear terms and a dissipation are small. After some transformations the following equations instead of Eq. (13) can be written:

$$
\left\{\begin{array}{l}
\ddot{x}+\omega_{x}^{2} x-q y+2 \varepsilon \eta_{x} \dot{x}+\varepsilon \gamma_{x}(x-y)^{3}=\varepsilon k \sin \Omega t \\
\ddot{y}+\omega_{y}^{2} y-\omega_{y}^{2} x+2 \varepsilon \eta_{y} \dot{y}-\varepsilon \gamma_{y}(x-y)^{3}=0,
\end{array}\right.
$$


(a)

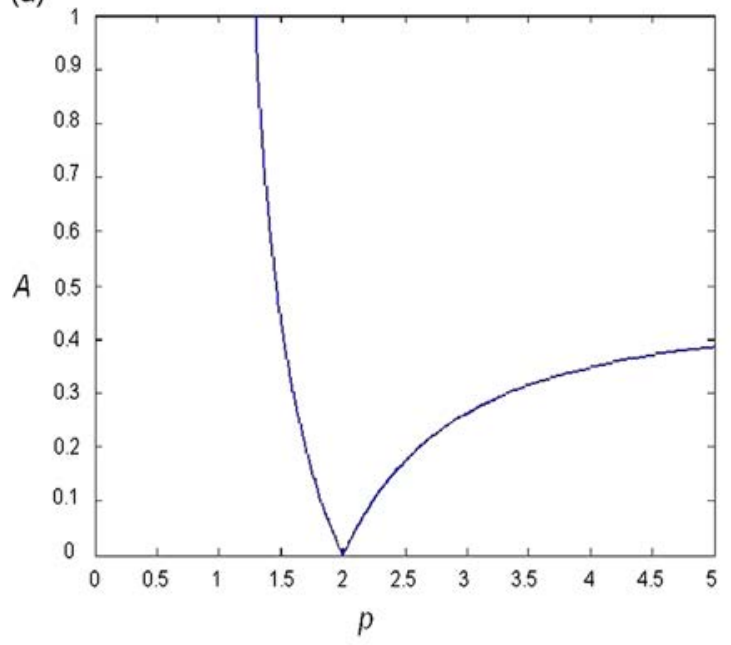

(b)

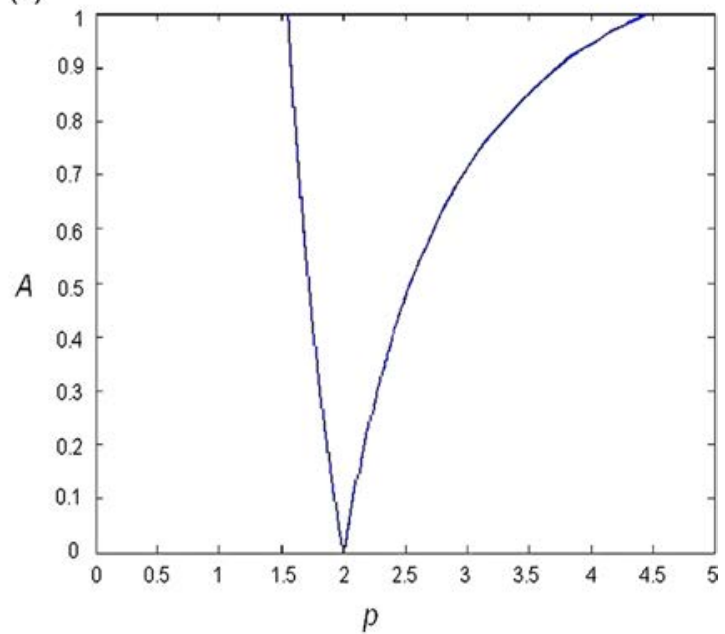

(c)

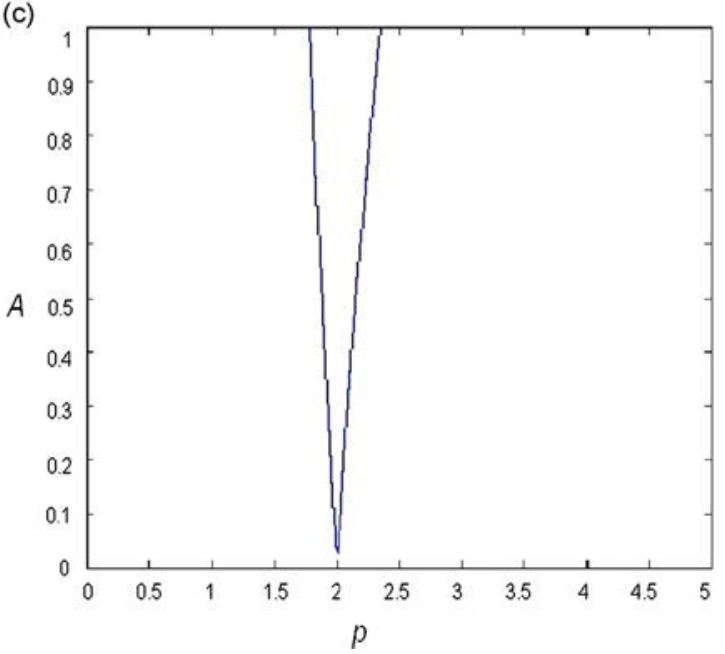

Fig. 7. Boundaries of the instability region on the place A, p at different values of $\tau$. (a) Boundaries at $\tau=0$; (b) boundaries at $\tau=50$; (c) boundaries at $\tau=100$.

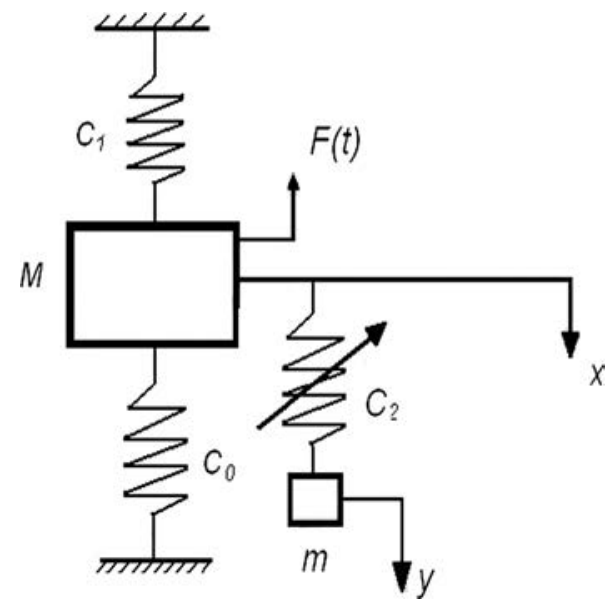

Fig. 8. System with the nonlinear absorber. 
where $\omega_{x}^{2}=\left(c+c_{2}\right) / M, 2 \eta_{x}=\beta / M, \gamma_{x}=\gamma / M, k=r / M, q=c_{2} / M, \omega_{y}^{2}=c_{2} / m, 2 \eta_{y}=\beta / m, \gamma_{y}=\gamma / m$. One considers, first of all, equations of the zero approximation of the perturbation procedure. Solving the corresponding eigenvalue problem, one obtains fundamental frequencies and eigenvectors $\chi_{1,2}=\left(\alpha_{1,2}, \beta_{1,2}\right)$ in the form:

$$
\begin{gathered}
\omega_{1,2}^{2}=\frac{\left(\omega_{x}^{2}+\omega_{y}^{2}\right) \mp \sqrt{\left(\omega_{x}^{2}-\omega_{y}^{2}\right)^{2}+4 \omega_{y}^{2} q}}{2}, \\
\left\{\begin{array}{l}
\alpha_{1,2}=1 \\
\beta_{1,2}=\frac{2 \omega_{y}^{2}}{\left(\omega_{y}^{2}-\omega_{x}^{2}\right) \pm \sqrt{\left(\omega_{x}^{2}-\omega_{y}^{2}\right)^{2}+4 \omega_{y}^{2} q}}
\end{array}\right. \\
\left(\operatorname{or} \beta_{1,2}=\frac{\left(\omega_{x}^{2}-\omega_{y}^{2}\right) \pm \sqrt{\left(\omega_{x}^{2}-\omega_{y}^{2}\right)^{2}+4 \omega_{y}^{2} q}}{2 q}\right) .
\end{gathered}
$$

A transfer to normal coordinates $\left\{\begin{array}{l}x=z_{1}+z_{2}, \\ y=\beta_{1} z_{1}+\beta_{2} z_{2} \text {. }\end{array}\right.$ gives the following:

$$
\left\{\begin{array}{l}
\ddot{z}_{1}+\omega_{1}^{2} z_{1}=\frac{\varepsilon \beta_{2}}{\beta_{2}-\beta_{1}}\left(-2 \eta_{x}\left(\dot{z}_{1}+\dot{z}_{2}\right)-\gamma_{x}\left(z_{1}+z_{2}-\beta_{1} z_{1}-\beta_{2} z_{2}\right)^{3}+k \sin \Omega t\right)-\frac{\varepsilon}{\beta_{2}-\beta_{1}}\left(-2 \eta_{y}\left(\beta_{1} \dot{z}_{1}+\beta_{2} \dot{z}_{2}\right)+\gamma_{y}\left(z_{1}+z_{2}-\beta_{1} z_{1}-\beta_{2} z_{2}\right)^{3}\right) \\
\ddot{z}_{2}+\omega_{2}^{2} z_{2}=\frac{\varepsilon}{\beta_{2}-\beta_{1}}\left(-2 \eta_{y}\left(\beta_{1} \dot{z}_{1}+\beta_{2} \dot{z}_{2}\right)+\gamma_{y}\left(z_{1}+z_{2}-\beta_{1} z_{1}-\beta_{2} z_{2}\right)^{3}\right)-\frac{\varepsilon \beta_{1}}{\beta_{2}-\beta_{1}}\left(-2 \eta_{x}\left(\dot{z}_{1}+\dot{z}_{2}\right)-\gamma_{x}\left(z_{1}+z_{2}-\beta_{1} z_{1}-\beta_{2} z_{2}\right)^{3}+k \sin \Omega t\right) .
\end{array}\right.
$$

If the external excitation and dissipation are absent, there are two NNMs in linearized equations of the system (17), which can be considered as localized modes in the first approximation of the perturbation procedure. Namely, there are two localized vibration modes. Namely, the first mode is presented as $z_{1}=z_{1}(t), z_{2} \equiv 0$, and the second mode is presented as $z_{1} \equiv 0, z_{2}=z_{2}(t)$. In the original coordinates of system (14) these modes can be considered respectively as non-localized mode, and as localized one. When the parameter $\beta_{1}=O(\varepsilon)$, or $\beta_{2}=O(\varepsilon)$, one has a localization on the coordinate $x$, and in the case when $\beta_{1}=O\left(\varepsilon^{-1}\right)$, or $\beta_{2}=O\left(\varepsilon^{-1}\right)$, on has a localization on the coordinate $y$. The condition $\beta_{1}=O(\varepsilon)\left(\right.$ or $\left.\beta_{2}=O(\varepsilon)\right)$ is guaranteed by smallness of the $\omega_{y}^{2}$, and the condition $\beta_{1}=O\left(\varepsilon^{-1}\right)\left(\right.$ or $\beta_{2}=O\left(\varepsilon^{-1}\right)$ ) is guaranteed by smallness of $q$, in accordance with the relation (16).

To analyze the external resonance on the natural frequency $\omega_{1}$, the detuning parameter $\Delta$ is introduced by the following equality: $\Omega^{2}=\omega_{1}^{2}+\varepsilon \Delta$. Then the multiple scales method is used with the following asymptotic series: $z_{1}=z_{10}\left(T_{0}, T_{1}, \ldots\right)+\varepsilon z_{11}\left(T_{0}, T_{1}, \ldots\right)+\ldots, z_{2}=z_{20}\left(T_{0}, T_{1}, \ldots\right)+\varepsilon z_{21}\left(T_{0}, T_{1}, \ldots\right)+\ldots$, where $T_{n}=\varepsilon^{n} t(n=0,1,2, \ldots)$. As a result, one obtains the following partial differential equations in two first approximations of the perturbation procedure:

$$
\begin{gathered}
\varepsilon^{0}:\left\{\begin{array}{l}
\frac{\partial^{2} z_{10}}{\partial T_{0}^{2}}+\Omega^{2} z_{10}=0 \\
\frac{\partial^{2} z_{20}}{\partial T_{0}^{2}}+\omega_{2}^{2} z_{20}=0
\end{array}\right. \\
\varepsilon^{1}:\left\{\begin{array}{l}
\frac{\partial^{2} z_{11}}{\partial T_{0}^{2}}+\Omega^{2} z_{11}=-2 \frac{\partial^{2} z_{10}}{\partial T_{0} \partial T_{1}}+\Delta z_{10}+\frac{1}{\beta_{2}-\beta_{1}}\left[\beta _ { 2 } \left(-2 \eta_{x} \frac{\partial\left(z_{10}+z_{20}\right)}{\partial T_{0}}-\gamma_{x}\left(z_{10}+z_{20}-\beta_{1} z_{10}-\beta_{2} z_{20}\right)^{3}\right.\right. \\
\left.\left.+k \sin \Omega T_{0}\right)+2 \eta_{y} \frac{\partial\left(\beta_{1} z_{10}+\beta_{2} z_{20}\right)}{\partial T_{0}}-\gamma_{y}\left(z_{10}+z_{20}-\beta_{1} z_{10}-\beta_{2} z_{20}\right)^{3}\right] \\
\frac{\partial^{2} z_{20}}{\partial T_{0}^{2}}+\omega_{2}^{2} z_{20}=-2 \frac{\partial^{2} z_{20}}{\partial T_{0} \partial T_{1}}+\frac{1}{\beta_{2}-\beta_{1}}\left[-2 \eta_{y} \frac{\partial\left(\beta_{1} z_{10}+\beta_{2} z_{20}\right)}{\partial T_{0}}+\gamma_{y}\left(z_{10}+z_{20}-\beta_{1} z_{10}-\beta_{2} z_{20}\right)^{3}\right. \\
\left.-\beta_{1}\left(-2 \eta_{x} \frac{\partial\left(z_{10}+z_{20}\right)}{\partial T_{0}}-\gamma_{x}\left(z_{10}+z_{20}-\beta_{1} z_{10}-\beta_{2} z_{20}\right)^{3}+k \sin \Omega T_{0}\right)\right] .
\end{array}\right.
\end{gathered}
$$

A solution of Eq. (18) can be written in the following form:

$$
\left\{\begin{array}{c}
z_{10}=C_{1} e^{i \Omega T_{0}}+\bar{C}_{1} e^{-i \Omega T_{0}} \\
z_{20}=C_{2} e^{i \omega_{2} T_{0}}+\bar{C}_{2} e^{-i \omega_{2} T_{0}}
\end{array}\right.
$$

Substituting the relations (20) to Eq. (19), one has the following condition of the secular generating terms elimination:

$$
\left\{\begin{array}{l}
-2 i \Omega_{2} \frac{\partial C_{1}}{\partial T_{0}}+(\Delta+i L) C_{1}-M C_{1}^{2} \bar{C}_{1}-P C_{1} C_{2} \bar{C}_{2}-i N=0 \\
-2 i \omega_{2} \frac{\partial C_{2}}{\partial T_{0}}+i S C_{2}+R C_{2}^{2} \bar{C}_{2}+T C_{2} C_{1} \bar{C}_{1}=0
\end{array}\right.
$$

where $L=2 \Omega /\left(\beta_{2}-\beta_{1}\right)\left(\beta_{1} \eta_{y}-\beta_{2} \eta_{x}\right), P=6\left(1-\beta_{1}\right)\left(1-\beta_{2}\right)^{2} /\left(\beta_{2}-\beta_{1}\right)\left(\beta_{2} \gamma_{x}+\gamma_{y}\right), \quad N=k \beta_{2} / 2\left(\beta_{2}-\beta_{1}\right), \quad M=3\left(1-\beta_{1}\right)^{3} /\left(\beta_{2}-\beta_{1}\right)$ $\left(\beta_{2} \gamma_{x}+\gamma_{y}\right), R=3\left(1-\beta_{2}\right)^{3} /\left(\beta_{2}-\beta_{1}\right)\left(\beta_{1} \gamma_{x}+\gamma_{y}\right), S=2 \omega_{2} /\left(\beta_{2}-\beta_{1}\right)\left(\beta_{1} \eta_{x}-\beta_{2} \eta_{y}\right), T=6\left(1-\beta_{2}\right)\left(1-\beta_{1}\right)^{2} /\left(\beta_{2}-\beta_{1}\right)\left(\beta_{1} \gamma_{x}+\gamma_{y}\right)$. 
Using a change of variables, $C_{1}=a_{1} e^{i b_{1}}, C_{2}=a_{2} e^{i b_{2}}$, one obtains the following equations with respect to amplitudes and phases of solutions:

$$
\left\{\begin{array}{l}
a_{1}^{\prime}=\frac{L}{2 \Omega} a_{1}-\frac{N}{2 \Omega} \cos b_{1} \\
b_{1}^{\prime}=-\frac{\Delta}{2 \Omega}+\frac{M}{2 \Omega} a_{1}^{2}+\frac{P}{2 \Omega} a_{2}^{2}+\frac{N}{2 \Omega a_{1}} \sin b_{1} \\
a_{2}^{\prime}=\frac{S}{2 \omega_{2}} a_{2} \\
b_{2}^{\prime}=-\frac{R}{2 \omega_{2}} a_{2}^{2}-\frac{T}{2 \omega_{2}} a_{1}^{2}
\end{array}\right.
$$

The following change of variables, $a_{1}=K \sin \psi, a_{2}=K \cos \psi$, permits to obtain the following reduced system:

$$
\left\{\begin{array}{l}
K^{\prime}=\frac{L}{2 \Omega} K \sin ^{2} \psi+\frac{S}{2 \omega_{2}} K \cos ^{2} \psi-\frac{N}{2 \Omega} \cos b_{1} \sin \psi \\
\psi^{\prime}=\left(\frac{L}{2 \Omega}-\frac{S}{2 \omega_{2}}\right) \sin \psi \cos \psi-\frac{N}{2 \Omega K} \cos \psi \cos b_{1} \\
b_{1}^{\prime}=-\frac{\Delta}{2 \Omega}+\frac{M}{2 \Omega} K^{2} \sin ^{2} \psi+\frac{P}{2 \Omega} K^{2} \cos ^{2} \psi+\frac{N}{2 \Omega K \sin \psi} \sin b_{1} \\
b_{2}^{\prime}=-\frac{R}{2 \omega_{2}} K^{2} \cos ^{2} \psi-\frac{T}{2 \omega_{2}} K^{2} \sin ^{2} \psi
\end{array}\right.
$$

where $K$ is an energy of the reduced system, $\psi$ is the arctangent of the ratio of amplitudes. An equation stated with respect to the difference of phases, $\varphi=b_{1}-b_{2}$, can be also obtained:

$$
\begin{gathered}
\varphi^{\prime}=-\frac{\Delta}{2 \Omega}+\frac{M}{2 \Omega} K^{2} \sin ^{2} \psi+\frac{P}{2 \Omega} K^{2} \cos ^{2} \psi+\frac{N}{2 \Omega K \sin \psi} \sin b_{1} \\
+\frac{R}{2 \omega_{2}} K^{2} \cos ^{2} \psi+\frac{T}{2 \omega_{2}} K^{2} \sin ^{2} \psi
\end{gathered}
$$

It is clear that due to the presence of external excitation in the system under consideration it is impossible to select the phase difference in the right hand part of Eq. (23). As a result, an analysis of the reduced system (23) is more complicated than in the preceding Section. But it is possible to investigate the second equation of the system (23) and Eq. (24) on their equilibrium solutions as functions of the energy $K$ and the detuning parameter $\Delta$. Relation $\sin \psi=0$ corresponds to localization of energy on coordinate $z_{2}$, and the relation $\cos \psi=0$ corresponds to localization on coordinate $z_{1}$.

In the case $\sin \psi=0$, a condition of existence of the equilibrium position in the second equation of the system (23) leads to relation $\cos b_{1}=0$. This equilibrium position of the reduced system is situated on the straight line $\psi=0$, and it leads to the equation $K^{\prime}=\left(S / 2 \omega_{2}\right) K$. It is possible to state prove that the quantity $S$ is negative for any parameters of the system, that is, the solution energy decreases. Thus, this equilibrium position corresponds to unstable vibrations.

In the case $\cos \psi=0$ the second equation of system (23) is an identity. This equilibrium position of the reduced system is situated on the straight line $\psi=\pi / 2$, and it leads to the equation $K^{\prime}=(L / 2 \Omega) K \pm(N / 2 \Omega) \cos b_{1}$. Here it is difficult to make some conclusion about evolution of the energy $K$. To obtain a result on stability of the solution it is necessary to analyze trajectories in the plane $\varphi, \psi$.

For the case when both $\sin \psi \neq 0$, and $\cos \psi \neq 0$, the equilibrium position for the second Eq. (23) exists if $\sin \psi=\left(N \omega_{2} / K\left(L \omega_{2}-S \Omega\right)\right) \cos b_{1}$. Thus, one has the inequality $\left|N \omega_{2} / K\left(L \omega_{2}-S \Omega\right)\right| \leq 1$; on the other hand, a condition of existence of $\cos b_{1}$ is $\left|K\left(L \omega_{2}-S \Omega\right) / N \omega_{2}\right| \leq 1$. So, this equilibrium position exists only for specific value of the energy, which meets the equality $\left|N \omega_{2} / K\left(L \omega_{2}-S \Omega\right)\right|=1$. From the Eq. (24) it is possible to obtain the following equation for the phase $b_{1}$ :

$$
\begin{aligned}
& \frac{L \omega_{2}-S \Omega}{2 \Omega \omega_{2}} \operatorname{tg}^{3} b_{1}+\left(\frac{-\Delta}{2 \Omega}+K^{2}\left(\frac{P}{2 \Omega}+\frac{R}{2 \omega_{2}}\right)\right) \operatorname{tg}^{2} b_{1}+\frac{L \omega_{2}-S \Omega}{2 \Omega \omega_{2}} \operatorname{tg}_{1} \\
& \quad+\left(\frac{-\Delta}{2 \Omega}+K^{2}\left(\frac{P}{2 \Omega}+\frac{R}{2 \omega_{2}}\right)\right)+\left(\frac{M-P}{2 \Omega}+\frac{T-R}{2 \omega_{2}}\right) \frac{N^{2} \omega_{2}^{2}}{\left(L \omega_{2}-S \Omega\right)^{2}}=0
\end{aligned}
$$

It is clear that in this case variables $\psi$ and $\varphi$ depend on the energy $K$, which decreases in time; so, the equilibrium position is not stationary. This state corresponds to vibrations, which are equivalent to NNM of coupled vibrations of the conservative sub-system (17). In the dissipative system this NNM is transient nonlinear normal vibration mode (TNNM). It appears only for some instant of time corresponding to specific value of the system energy that responds to the presented above equality and to value of the phase $b_{1}$ determined from the Eq. (25). In the vicinity of this time the system motions are close to this transient vibration mode. If the initial energy is small, and the energy value corresponding to the mode of coupled vibrations, is not reached, this transient vibration mode is not realized.

One integrates Eq. (13) by the Runge-Kutta method, choosing the initial values on the interval $0 \leq \psi(0) \leq \pi / 2$ for $K(0)=0.5, c_{0}=0.3, c_{1}=0.1, c_{2}=0.2, M=1.9, m=0.4, \beta=0.2, \gamma=0.3, r=0.01$ and $\Delta=0,1$. The dependence $\varphi(\psi)$ is shown in Fig. 9.

One can see in Fig. 9 that trajectories which initially are close to the straight line $\psi=0$, then approach the straight line $\psi=\pi / 2$; it means that vibrations, localized on $z_{1}$, are stable near the resonance, whereas vibrations, localized on $z_{2}$, lose stability. Simultaneously some trajectories tend to an equilibrium position corresponding to the mode of coupled vibrations, and remain near this state until this state exists. So, an appearance of the attractive TNNM of coupled vibrations near 


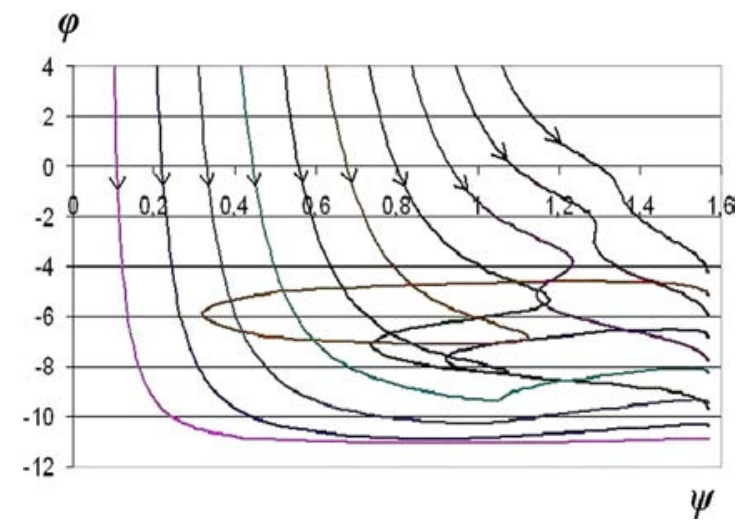

Fig. 9. Forced resonance on the first fundamental frequency. Dependence $\varphi(\psi)$.

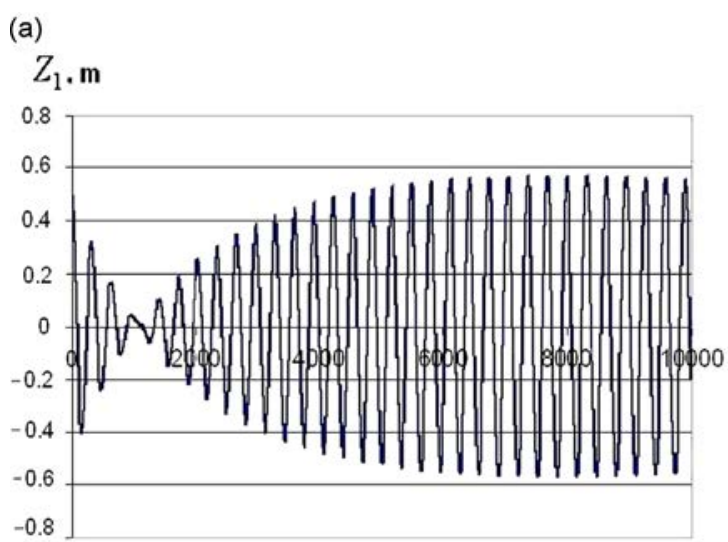

$t \cdot 20, \mathrm{c}$ (b)

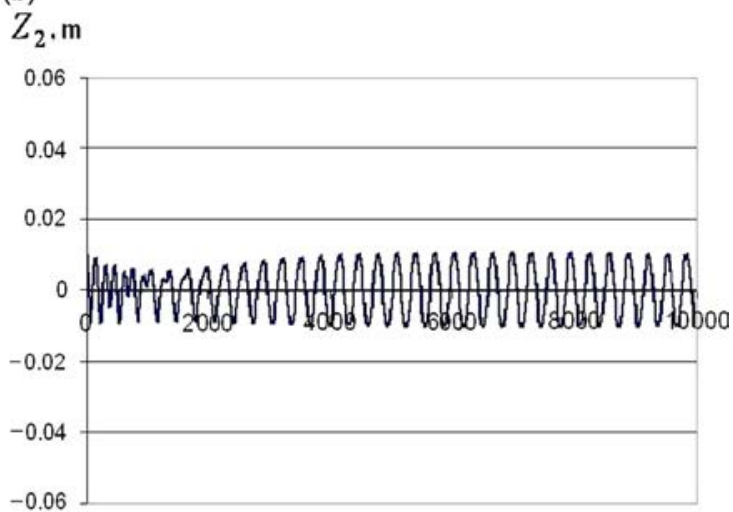

$t * 20, \mathrm{c}$

(c)

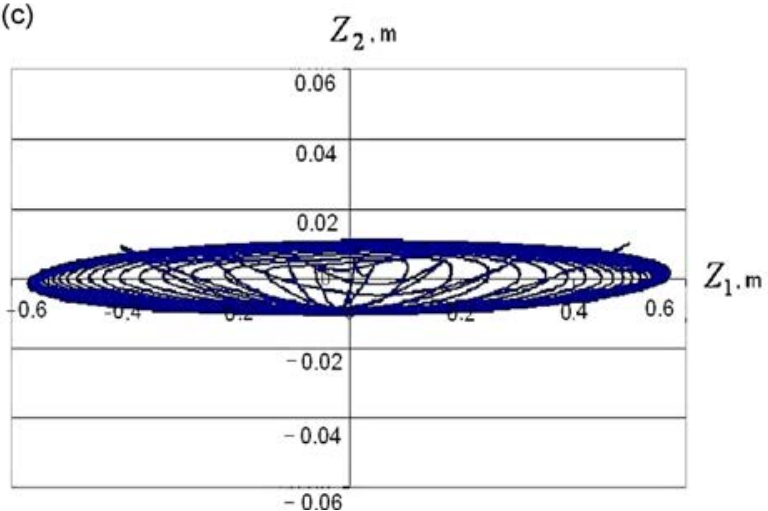

Fig. 10. Stable mode of localization on coordinate $z_{1}$. (a) Dependence $z_{1}(t)$; (b) dependence $z_{2}(t)$; (c) the variables $z_{1}$ and $z_{2}$ in configuration space.

resonance for values of $\psi$ and $b_{1}$, which satisfy to Equation (36), is possible. In some time the mode of coupled vibrations does not exist, and motions of the system tend to the stable localized mode.

Figs. 10,11 illustrate a behavior of the system (17) near the resonance. Here numerical integration by the Runge-Kutta method of the 4-th order is used on the interval $t \in[0 ; 500]$ for $\Omega=0.3996, c_{0}=0.3, c_{1}=0.1, c_{2}=0.2, M=1.9$, $m=0.4, \beta=0.2, \gamma=0.2, r=0.15, \varepsilon=0.1, \Delta=-0.01$. The initial values $z_{1}(0)=0.5, z_{1}^{\prime}(0)=0, z_{2}(0)=0.01, z_{2}^{\prime}(0)=0$ are chosen for the mode of localization on the coordinate $z_{1}$, and initial values $z_{1}(0)=0.01, z_{1}^{\prime}(0)=0, z_{2}(0)=0.5, z_{2}^{\prime}(0)=0$ are chosen for the mode of localization on coordinate $z_{2}$. 


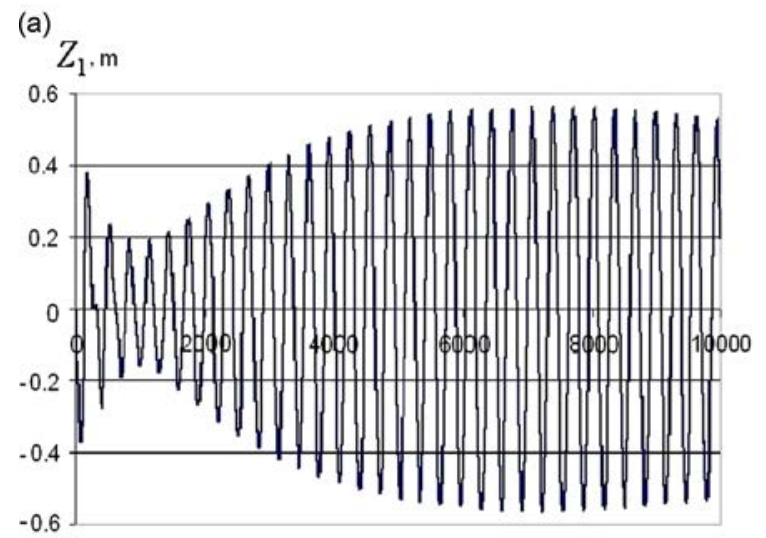

(b)

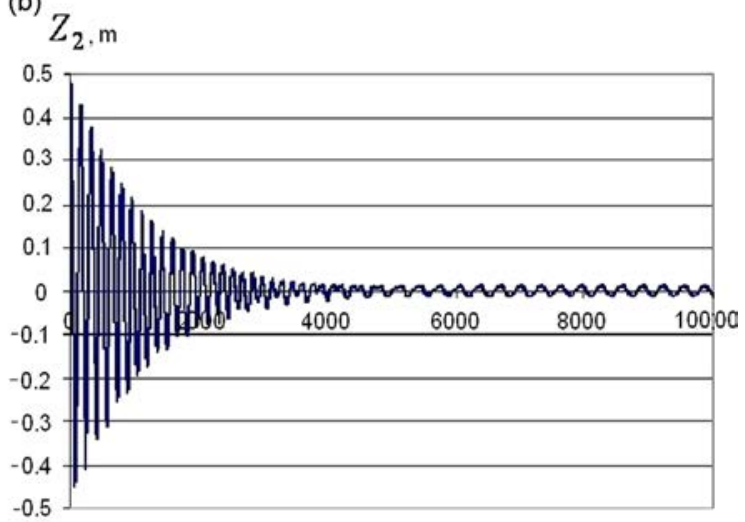

$t$ '20, c

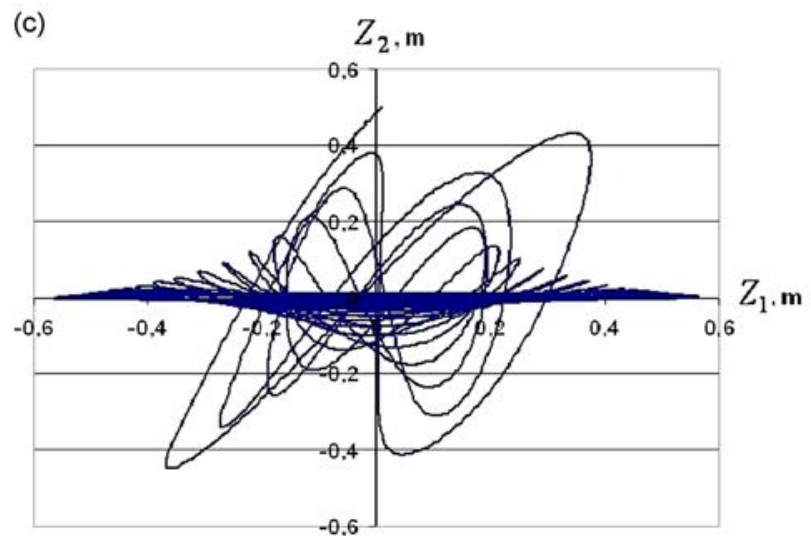

Fig. 11. Unstable mode of localization on coordinate $z_{2}$. (a) Dependence $z_{1}(t)$; (b) dependence $z_{2}(t)$; (c) the variables $z_{1}$ and $z_{2}$ in configuration space.

In Fig. 10 dependences of the coordinates $z_{1}$ and $z_{2}$ versus time and in the configuration place are presented for the mode of localization on the coordinate $z_{1}$. A numerical simulation confirms that the localized mode is stable in the vicinity of resonance. Amplitudes of the variable $z_{2}$ are essentially smaller than ones of the variable $z_{1}$. Dependences of the coordinates $z_{1}$ and $z_{2}$ on time and in the configuration space for the mode of localization on the coordinate $z_{2}$ are presented in Fig. 11 . Numerical simulation shows that this localized mode is unstable in the vicinity of resonance. Amplitudes of the variable $z_{1}$ are compared with ones of the variable $z_{2}$. Moreover, one can see (Fig. 11a,b) that near the time instant $t=50$ motions of the system approach to the TNNM of coupled vibrations. It corresponds to results obtained by analysis of the reduced system. If the time increases, a transfer to the stable mode of localization on the coordinate $z_{1}$ takes place.

Thus, results of numerical simulation confirm results obtained by analysis of the reduced system for the case of external resonance on the frequency $\omega_{1}$.

\subsection{Investigation of external resonance on the second fundamental frequency}

To analyze external resonance on the natural frequency $\omega_{2}$, one introduces the detuning parameter $\Delta$ by an equality $\Omega^{2}=\omega_{2}^{2}+\varepsilon \Delta$.

Applying to the system (13) in the vicinity of resonance the approach described in the sub-Section 3.1, one can obtain from a condition of the secular generating terms elimination the following system stated with respect to amplitudes and phases of the solution:

$$
\left\{\begin{array}{l}
a_{1}^{\prime}=\frac{\tilde{L}}{2 \omega_{1}} a_{1} \\
b_{1}^{\prime}=\frac{M}{2 \omega_{1}} a_{1}^{2}+\frac{P}{2 \omega_{1}} a_{2}^{2} \\
a_{2}^{\prime}=\frac{\tilde{S}}{2 \Omega} a_{2}+\frac{N}{2 \Omega} \cos b_{2} \\
b_{2}^{\prime}=-\frac{\Delta}{2 \Omega}-\frac{R}{2 \Omega} a_{2}^{2}-\frac{T}{2 \Omega} a_{1}^{2}-\frac{N}{2 \Omega a_{2}} \sin b_{2}
\end{array}\right.
$$


where $\tilde{L}=\left(2 \omega_{1} /\left(\beta_{2}-\beta_{1}\right)\right)\left(\beta_{1} \eta_{y}-\beta_{2} \eta_{x}\right), \quad P=\left(6\left(1-\beta_{1}\right)\left(1-\beta_{2}\right)^{2} /\left(\beta_{2}-\beta_{1}\right)\right)\left(\beta_{2} \gamma_{x}+\gamma_{y}\right), \quad N=k \beta_{2} / 2\left(\beta_{2}-\beta_{1}\right), \quad M=\left(3\left(1-\beta_{1}\right)^{3} /\left(\beta_{2}-\right.\right.$ $\left.\left.\beta_{1}\right)\right)\left(\beta_{2} \gamma_{x}+\gamma_{y}\right), R=\left(3\left(1-\beta_{2}\right)^{3} /\left(\beta_{2}-\beta_{1}\right)\right)\left(\beta_{1} \gamma_{x}+\gamma_{y}\right), \tilde{S}=\left(2 \Omega /\left(\beta_{2}-\beta_{1}\right)\right)\left(\beta_{1} \eta_{x}-\beta_{2} \eta_{y}\right), T=\left(6\left(1-\beta_{2}\right)\left(1-\beta_{1}\right)^{2} /\left(\beta_{2}-\beta_{1}\right)\right)\left(\beta_{1} \gamma_{x}+\gamma_{y}\right)$.

Then the change of variables, $a_{1}=K \sin \psi, a_{2}=K \cos \psi$, permits to obtain the following reduced system:

$$
\left\{\begin{array}{l}
K^{\prime}=\frac{\tilde{L}}{2 \omega_{1}} K \sin ^{2} \psi+\frac{\tilde{S}}{2 \Omega} K \cos ^{2} \psi+\frac{N}{2 \Omega} \cos b_{2} \cos \psi \\
\psi^{\prime}=\left(\frac{\tilde{L}}{2 \omega_{1}}-\frac{\tilde{S}}{2 \Omega}\right) \sin \psi \cos \psi-\frac{N}{2 \Omega K} \sin \psi \cos b_{2} \\
b_{1}^{\prime}=\frac{M}{2 \omega_{1}} K^{2} \sin ^{2} \psi+\frac{P}{2 \omega_{1}} K^{2} \cos ^{2} \psi \\
b_{2}^{\prime}=-\frac{\Delta}{2 \Omega}-\frac{R}{2 \Omega} K^{2} \cos ^{2} \psi-\frac{T}{2 \Omega} K^{2} \sin ^{2} \psi-\frac{N}{2 \Omega K \cos \psi} \sin b_{2},
\end{array}\right.
$$

where $K$ is the system energy, $\psi$ is the arctangent of the ratio of amplitudes. Equation for the difference of phases, $\varphi=b_{1}-b_{2}$, can be obtained as

$$
\begin{gathered}
\varphi^{\prime}=\frac{M}{2 \omega_{1}} K^{2} \sin ^{2} \psi+\frac{P}{2 \omega_{1}} K^{2} \cos ^{2} \psi+\frac{\Delta}{2 \Omega}+\frac{R}{2 \Omega} K^{2} \cos ^{2} \psi \\
+\frac{T}{2 \Omega} K^{2} \sin ^{2} \psi+\frac{N}{2 \Omega K \cos \psi} \sin b_{2}
\end{gathered}
$$

It is impossible to select the phase difference in the right hand part of the system (27). The second equation of the system (27) and Eq. (28) are investigated on their equilibrium solutions as functions of the energy $K$ and the detuning parameter $\Delta$.

In the case $\sin \psi=0$ the second equation of the system (27) is an identity. This relation corresponds to localization of the system energy on the coordinate $z_{2}$. It is on the straight line $\psi=0$; corresponding equation of the energy is $K^{\prime}=(\tilde{S} / 2 \Omega) K \pm(N / 2 \Omega) \cos b_{2}$. Here it is difficult to make some conclusion about evolution of the energy $K$. It is necessary to analyze additionally trajectories in the system configuration space.

In the case $\cos \psi=0$, a condition of existence of equilibrium position in the second equation of the system (27) gives the relation $\cos b_{2}=0$. This equilibrium position of the reduced system is situated on the straight line $\psi=\pi / 2$, and it leads to the equation $K^{\prime}=\left(\tilde{L} / 2 \omega_{2}\right) K$. It is possible to state that the quantity $\tilde{L}$ is negative for any parameters of the system. This, the solution is not attractive, and this equilibrium position corresponds to unstable vibrations.

For the case, when $\sin \psi \neq 0$, and $\cos \psi \neq 0$, equilibrium positions of the second Eq. (27) requires the following relation: $\cos \psi=\left(N \omega_{1} / K\left(\tilde{L} \Omega-\tilde{S} \omega_{1}\right)\right) \cos b_{2}$. From here two inequalities are derived: $\left|N \omega_{1} / K\left(\tilde{L} \Omega-\tilde{S} \omega_{1}\right)\right| \leq 1$, and $\left|K\left(\tilde{L} \Omega-\tilde{S} \omega_{1}\right) / N \omega_{1}\right| \leq 1$. As a result, this equilibrium position exists only for the energy value which meets the following equality: $\left|N \omega_{1} / K\left(\tilde{L} \Omega-\tilde{S} \omega_{1}\right)\right|=1$. Besides, one has from Eq. (28) the following equation for the phase $b_{2}$ :

$$
\begin{aligned}
& \frac{\tilde{L} \Omega-\tilde{S} \omega_{1}}{2 \Omega \omega_{1}} \operatorname{tg}^{3} b_{2}+\left(\frac{\Delta}{2 \Omega}+K^{2}\left(\frac{T}{2 \Omega}+\frac{M}{2 \omega_{1}}\right)\right) \operatorname{tg}^{2} b_{2}+\frac{\tilde{L} \Omega-\tilde{S} \omega_{1}}{2 \Omega \omega_{1}} \operatorname{tg}_{2} \\
& \quad+\left(\frac{\Delta}{2 \Omega}+K^{2}\left(\frac{T}{2 \Omega}+\frac{M}{2 \omega_{1}}\right)\right)+\left(\frac{R-T}{2 \Omega}+\frac{P-M}{2 \omega_{1}}\right) \frac{N^{2} \omega_{1}^{2}}{\left(\tilde{L} \Omega-\tilde{S} \omega_{1}\right)^{2}}=0
\end{aligned}
$$

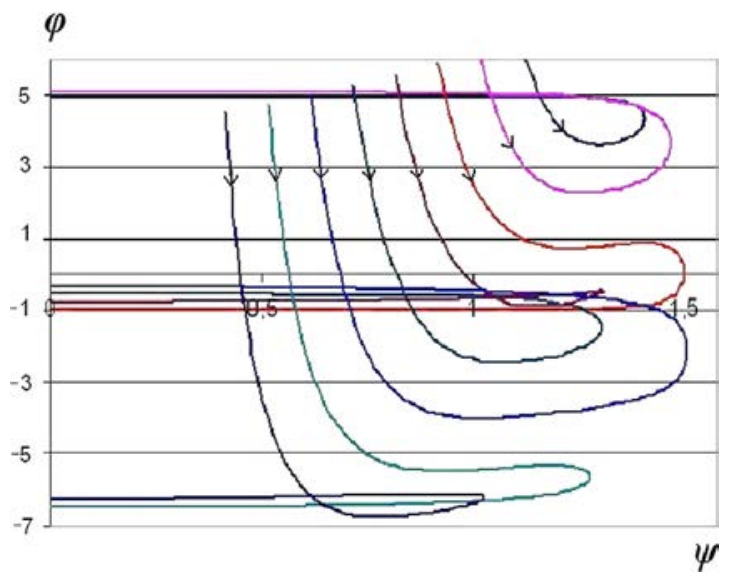

Fig. 12. Forced resonance on the second fundamental frequency. Dependence $\varphi(\psi)$. 


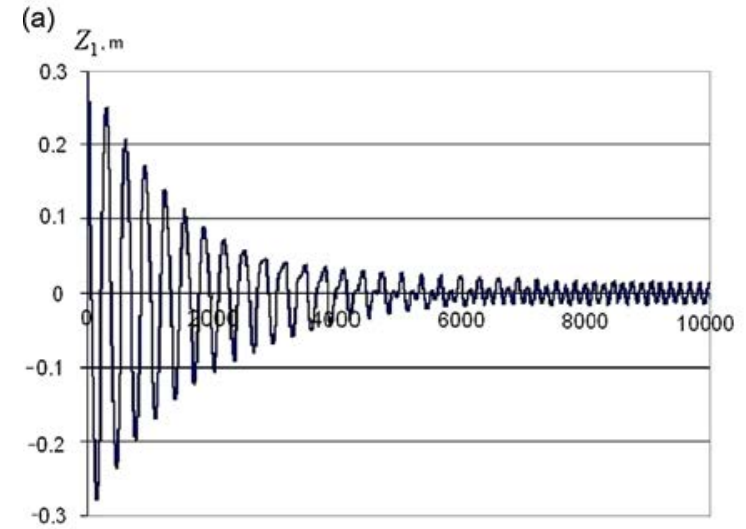

$t \cdot 20, \mathrm{c}$ (b)

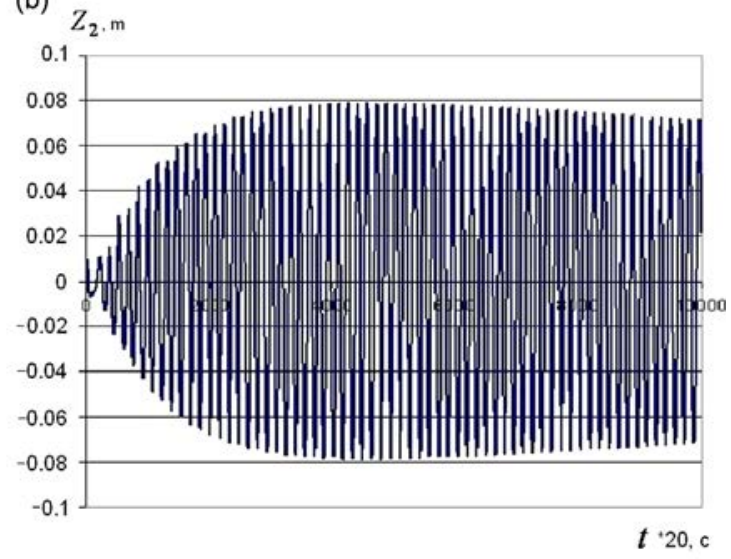

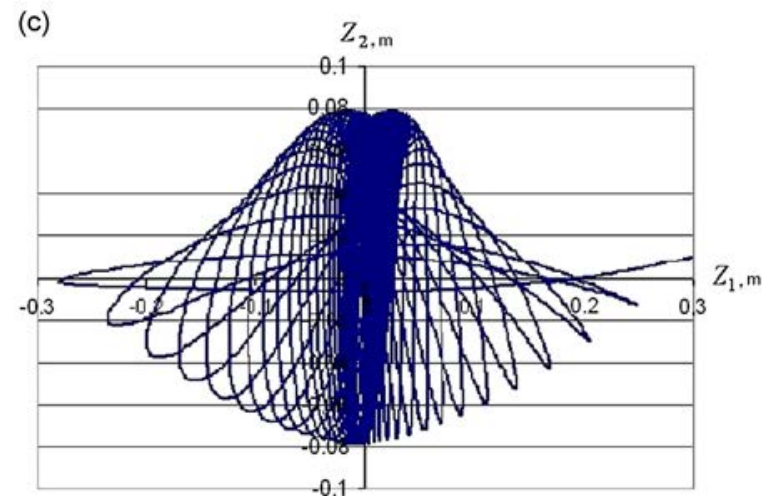

Fig. 13. Unstable mode of localization on coordinate $z_{1}$. (a) Dependence $z_{1}(t)$; (b) dependence $z_{2}(t)$; (c) the variables $z_{1}$ and $z_{2}$ in configuration space.

In this case the equilibrium position is not the stationary one. The transient nonlinear normal mode of the dissipative system appears in the time instant when the system energy corresponds to the presented above equality and value of the phase $b_{2}$ responds to the Eq. (29). In the vicinity of this instant of time the system motion is close to this vibration mode. If the initial energy is small the TNNM is not realized. Note that it corresponds to NNM of coupled vibrations of the conservative subsystem (17).

One integrates the Eq. (29) by the Runge-Kutta method of the 4-th order, choosing the initial values on the interval $0 \leq \psi(0) \leq \pi / 2$ for $K(0)=0.5, c_{0}=0.3, c_{1}=0.1, c_{2}=0.2, M=1.9, m=0.4, \beta=0.2, \gamma=0.3, r=0.01$ and $\Delta=0.1$. The dependence $\varphi(\psi)$ is shown in Fig. 12. One can see that at the beginning trajectories withdraw from the straight line $\psi=0$, which corresponds to vibrations which are localized on the coordinate $z_{2}$. The localized mode is unstable for current levels of energy. Then trajectories approach equilibrium position corresponding to TNNM of coupled vibrations and do a loop near this position. Note that this position moves in the place $\varphi, \psi$. When the energy decreases, and this position vanishes, trajectories withdraw from it and during some time approach the straight line $\psi=\pi / 2$, but do not remain near the last position because it correspond to unstable vibration mode localized on the coordinate $z_{1}$. Then the localized on $z_{2}$ vibrations became stable, and trajectories approach the straight line $\psi=0$.

One illustrates a behavior of the system (17) near resonance using the numerical integration by the Runge-Kutta method on the interval $t \in[0 ; 500]$ for $\Omega=0.80877, c_{0}=0.3, c_{1}=0.1, c_{2}=0.2, M=1.9, m=0.4, \beta=0.2, \gamma=0.2, r=0.15, \varepsilon=0.1$, $\Delta=-0.01$ and initial values $z_{1}(0)=0.3, z_{1}^{\prime}(0)=0, z_{2}(0)=0.01, z_{2}^{\prime}(0)=0$ for the mode of localization on the coordinate $z_{1}$, and initial values $z_{1}(0)=0.01, z_{1}^{\prime}(0)=0, z_{2}(0)=0.3, z_{2}^{\prime}(0)=0$ for the mode of localization on coordinate $z_{2}$.

In Fig. 13 dependences of the coordinates $z_{1}$ and $z_{2}$ on time and in the configuration space for the mode of localization on the coordinate $z_{1}$ are presented. Numerical simulation shows that the localized mode doesn't lose stability in the beginning of the process. Instability develops slowly. Transfer from the localization on $z_{1}$ to the stable vibration mode of localization on $z_{2}$ is shown in Fig. 13c. In Fig. 14 dependences of variables $z_{1}$ and $z_{2}$ in time and in the configuration space for the mode of localization on the coordinate $z_{2}$ are presented. Numerical simulation shows that this localized mode loses stability in the vicinity of resonance for some initial time interval. In times close to the value $t=70$ motion of the system is close to TNNM of coupled vibrations. Then one can observe a stabilization of the vibration mode which is localized on the coordinate $z_{2}$. 


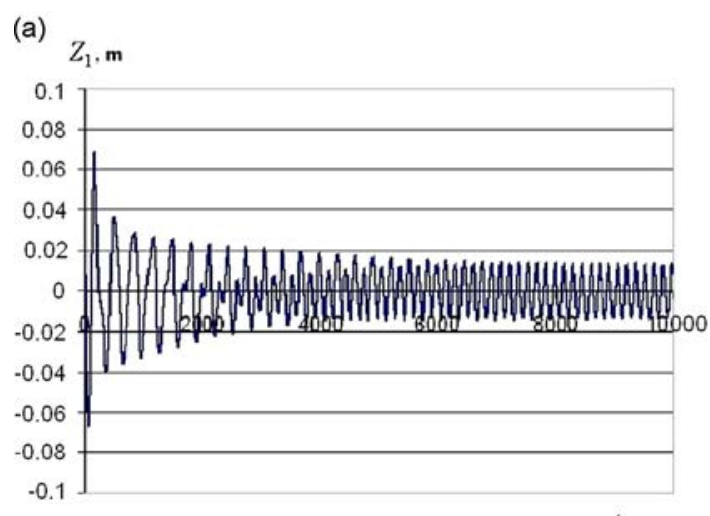

$t * 20, c$ (b)

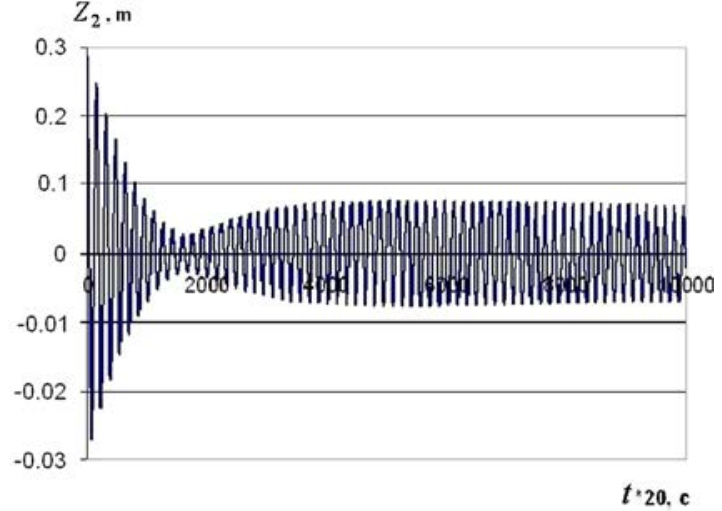

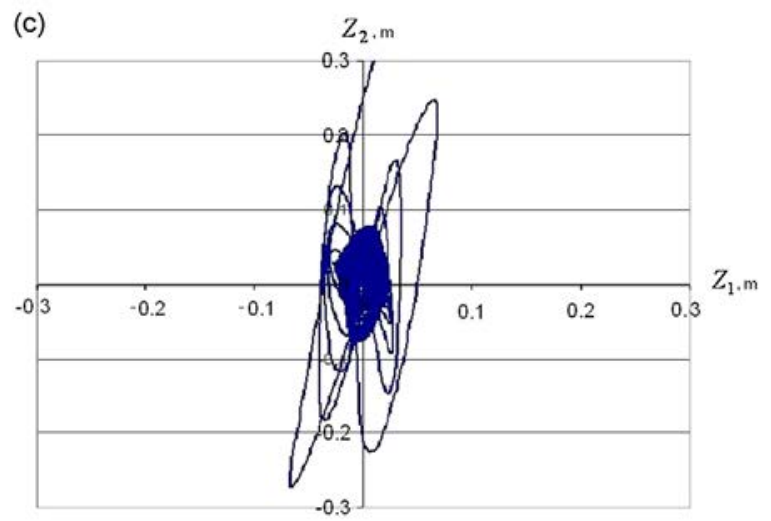

Fig. 14. Mode of localization on coordinate $z_{2}$. (a) Dependence $z_{1}(t)$; (b) dependence $z_{2}(t)$; (c) the variables $z_{1}$ and $z_{2}$ in configuration space.

Thus, results of numerical simulation fully confirm results obtained by analysis of the reduced system in region of the external resonance on the frequency $\omega_{2}$.

\subsection{Investigation of simultaneous external and internal resonances}

One considers a case of simultaneous external and internal resonances. The following detuning of the natural frequencies $\omega_{1}$ and $\omega_{2}$, and external frequency $\Omega$ are introduced: $\omega_{1}^{2}=\omega_{2}^{2}+\varepsilon \sigma, \Omega^{2}=\omega_{1}^{2}+\varepsilon \Delta_{1}, \Omega^{2}=\omega_{2}^{2}+\varepsilon \Delta_{2}, \Delta_{2}=\Delta_{1}+\sigma$. Then the standard expansions of the multiple-scale method are used.

Elimination of secular generating terms leads to the next system stated with respect to amplitudes and phases of solutions:

$\left\{\begin{array}{l}a_{1}^{\prime}=\frac{L_{1}}{2} a_{1}+\frac{N_{1}}{2} a_{2} \cos \left(b_{1}-b_{2}\right)-\frac{P_{1}}{2 \Omega}\left[-B a_{2}^{2} a_{1} \sin \left(2 b_{1}-2 b_{2}\right)+(E-D) a_{1}^{2} a_{2} \sin \left(b_{1}-b_{2}\right)-F a_{2}^{3} \sin \left(b_{1}-b_{2}\right)\right]-\frac{\delta}{2 \Omega} \cos b_{1} \\ b_{1}^{\prime}=-\frac{\Delta_{1}}{2 \Omega}-\frac{N_{1}}{2 a_{1}} a_{2} \sin \left(b_{1}-b_{2}\right)+\frac{P_{1}}{2 \Omega a_{1}}\left[A a_{1}^{3}+B a_{2}^{2} a_{1} \cos \left(2 b_{1}-2 b_{2}\right)+(E+D) a_{1}^{2} a_{2} \cos \left(b_{1}-b_{2}\right)+F a_{2}^{3} \cos \left(b_{1}-b_{2}\right)+G a_{1} a_{2}^{2}\right]+\frac{\delta}{2 \Omega a_{1}} \sin b_{1} \\ a_{2}^{\prime}=\frac{L_{2}}{2} a_{2}+\frac{N_{2}}{2} a_{1} \cos \left(b_{1}-b_{2}\right)-\frac{P_{2}}{2 \Omega}\left[-A a_{1}^{3} \sin \left(b_{1}-b_{2}\right)+(B-G) a_{2}^{2} a_{1} \sin \left(b_{1}-b_{2}\right)+E a_{1}^{2} a_{2} \sin \left(2 b_{1}-2 b_{2}\right)\right]+\frac{\delta}{2 \Omega} \cos b_{2} \\ b_{2}^{\prime}=-\frac{\Delta_{2}}{2 \Omega}+\frac{N_{2}}{2 a_{2}} a_{1} \sin \left(b_{1}-b_{2}\right)-\frac{P_{2}}{2 \Omega a_{2}}\left[A a_{1}^{3} \cos \left(b_{1}-b_{2}\right)+(B+G) a_{2}^{2} a_{1} \cos \left(b_{1}-b_{2}\right)+D a_{1}^{2} a_{2}+E a_{1}^{2} a_{2} \cos \left(2 b_{1}-2 b_{2}\right)+F a_{2}^{3}\right]-\frac{\delta}{2 \Omega a_{2}} \sin b_{2},\end{array}\right.$

where $\delta=\beta_{2} k / 2\left(\beta_{2}-\beta_{1}\right), \quad L_{1}=\left(2 /\left(\beta_{2}-\beta_{1}\right)\right)\left(\beta_{1} \eta_{y}-\beta_{2} \eta_{x}\right), \quad N_{1}=\left(2 /\left(\beta_{2}-\beta_{1}\right)\right)\left(\beta_{2} \eta_{y}-\beta_{2} \eta_{x}\right), \quad P_{1}=\left(1 /\left(\beta_{2}-\beta_{1}\right)\right)\left(\beta_{2} \gamma_{x}+\gamma_{y}\right), \quad L_{2}=$ $\left(2 /\left(\beta_{2}-\beta_{1}\right)\right)\left(\beta_{1} \eta_{x}-\beta_{2} \eta_{y}\right), \quad N_{2}=\left(2 /\left(\beta_{2}-\beta_{1}\right)\right)\left(\beta_{1} \eta_{x}-\beta_{1} \eta_{y}\right), P_{2}=\left(1 /\left(\beta_{2}-\beta_{1}\right)\right)\left(\beta_{1} \gamma_{x}+\gamma_{y}\right), A=1+3 \beta_{1}^{2}-3 \beta_{1}-\beta_{1}^{3}, F=1+3 \beta_{2}^{2}-3 \beta_{2}-$ $\beta_{2}^{3}, \quad B=1+\beta_{2}^{2}-2 \beta_{2}-\beta_{1}-\beta_{2}^{2} \beta_{1}+2 \beta_{2} \beta_{1}, \quad D=2-2 \beta_{2}-4 \beta_{1}+4 \beta_{1} \beta_{2}+2 \beta_{1}^{2}-2 \beta_{1}^{2} \beta_{2}, \quad G=2-2 \beta_{1}-4 \beta_{2}+4 \beta_{1} \beta_{2}+2 \beta_{2}^{2}-2 \beta_{2}^{2} \beta_{1}, \quad E=$ $1+\beta_{1}^{2}-2 \beta_{1}-\beta_{2}-\beta_{1}^{2} \beta_{2}+2 \beta_{2} \beta_{1}$. 
Change of variables, $a_{1}=K \sin \psi, a_{2}=K \cos \psi$, permits to obtain the following reduced system:

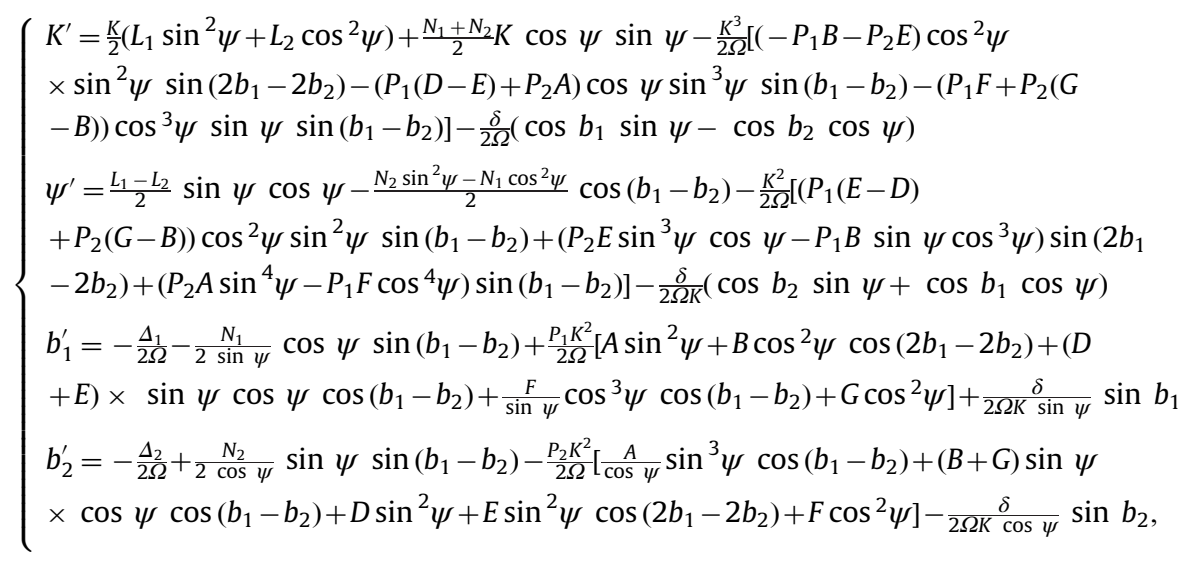

where $K$ is the system energy, $\psi$ is the arctangent of the amplitudes ratio. An equation with respect to the difference of phases, $\varphi=b_{1}-b_{2}$ can be written as

$$
\begin{gathered}
\varphi^{\prime}=-\frac{\Delta_{1}}{2 \Omega}+\frac{\Delta_{2}}{2 \Omega}-\left(\frac{N_{1}}{2 \sin \psi} \cos \psi+\frac{N_{2}}{2 \cos \psi} \sin \psi\right) \sin \left(b_{1}-b_{2}\right)+\frac{P_{1} K^{2}}{2 \Omega}\left[A \sin ^{2} \psi+B \cos ^{2} \psi\right. \\
\left.\times \cos \left(2 b_{1}-2 b_{2}\right)+(D+E) \sin \psi \cos \psi \cos \left(b_{1}-b_{2}\right)+\frac{F}{\sin \psi} \cos ^{3} \psi \cos \left(b_{1}-b_{2}\right)+G \cos ^{2} \psi\right] \\
+\frac{P_{2} K^{2}}{2 \Omega}\left[\frac{A}{\cos \psi} \sin ^{3} \psi \cos \left(b_{1}-b_{2}\right)+(B+G) \sin \psi \cos \psi \cos \left(b_{1}-b_{2}\right)+D \sin ^{2} \psi\right. \\
\left.\left.+E \sin ^{2} \psi \sin ^{2} \psi \cos \left(2 b_{1}-2 b_{2}\right)+F \cos ^{2} \psi\right]+\frac{\delta}{2 \Omega K}\left(\frac{\sin b_{1}}{\sin \psi}+\frac{\sin b_{2}}{\cos \psi}\right)\right) .
\end{gathered}
$$

We will analyze the second equation of the system (31) and Eq. (32) on their equilibrium states as functions of the energy $K$ and the detuning parameter $\Delta$.

Relation $\sin \psi=0$ corresponds to the localized mode of the system (17) with localization on the coordinate $z_{2}$; relation $\cos \psi=0$ corresponds to the localized mode of the system (17) with localization on the coordinate $z_{1}$.

For relation $\sin \psi=0$, a condition of equilibrium for the second eq. (31) gives us the following equality: $\Omega K N_{1} \cos \varphi+K^{3} P_{1} F \sin \varphi= \pm \delta \cos b_{1}$. It can be rewritten as $\sqrt{\Omega^{2} K^{2} N_{1}^{2}+K^{6} P_{1}^{2} F^{2}} \sin (\varphi+\lambda)= \pm \delta$ cos $b_{1}$, where $\lambda=\operatorname{arctg}\left(N_{1} \Omega / K^{2} P_{1} F\right)$. Conditions of existing of the functions $\sin (\varphi+\lambda)$ and $\cos b_{1}$ give two inequalities, respectively, $\left|\sqrt{\Omega^{2} K^{2} N_{1}^{2}+K^{6} P_{1}^{2} F^{2}} / \delta\right| \leq 1$ and $\left|\delta / \sqrt{\Omega^{2} K^{2} N_{1}^{2}+K^{6} P_{1}^{2} F^{2}}\right| \geq 1$. Thus, one has a condition of existing of the vibration mode, localized on the coordinate $z_{2}$, in the following form: $\left|\sqrt{\Omega^{2} K^{2} N_{1}^{2}+K^{6} P_{1}^{2} F^{2}} / \delta\right|=1$. From the last equality one can obtain specific values of energy when this transient mode exists. Equation $K^{\prime}=\frac{L_{2}}{2} K \mp \delta \cos b_{2}$ corresponds to the TNNM. From this equation it is difficult to draw some definite conclusion about the energy $K$ evolution. It is necessary to analyze trajectories in the system configuration space.

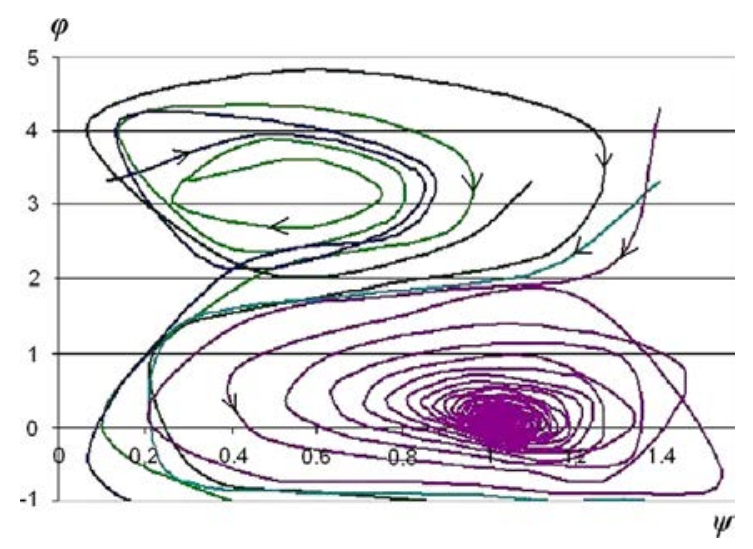

Fig. 15. Case of simultaneous external and internal resonances. Dependence $\varphi(\psi)$. 
In the case $\cos \psi=0$, a condition of existence of equilibrium position in the second equation of the system (31) gives us the following relation: $\Omega K N_{2} \cos \varphi+K^{3} P_{2} A \sin \varphi= \pm \delta \cos b_{2}$. One has from here the equality $\sqrt{\Omega^{2} K^{2} N_{2}^{2}+K^{6} P_{2}^{2} A^{2}} \sin (\varphi+\lambda)= \pm \delta \cos b_{2}$, where $\lambda=\operatorname{arctg}\left(N_{2} \Omega / K^{2} P_{2} A\right)$. Conditions of existence of the corresponding trigonometric functions give the next inequalities: $\left|\sqrt{\Omega^{2} K^{2} N_{2}^{2}+K^{6} P_{2}^{2} A^{2}} / \delta\right| \leq 1$, and $\left|\delta / \sqrt{\Omega^{2} K^{2} N_{2}^{2}+K^{6} P_{2}^{2} A^{2}}\right| \geq 1$. Thus, a condition of existence of the vibration localization on the coordinate $z_{1}$ is the following: $\left|\sqrt{\Omega^{2} K^{2} N_{2}^{2}+K^{6} P_{2}^{2} A^{2}} / \delta\right|=1$. We can obtain from here some specific values of the system energy. The equation $K^{\prime}=\left(L_{1} / 2\right) K \pm \delta \cos b_{1}$ describes a change of energy for this TNNM. Here, due to a presence of the function $b_{1}(K)$ in this equation, it is difficult to draw a conclusion on the mode stability; it is necessary to analyze trajectories in the system configuration space too.

Thus, we can conclude that both localized modes can be realized in the resonance region only near such time instants when specific values of the energy are reached. Other time the energy localization in the system (17) is not observed, that is the system vibrations are coupled ones $(\sin \psi \neq 0, \cos \psi \neq 0$ ).

The system (31) has been integrated by the Runge-Kutta method. The initial values are chosen on the interval $0 \leq \psi(0) \leq \pi / 2$ for $K(0)=0.08, c_{0}=0.9, c_{1}=1.05, c_{2}=0.05, M=40, m=1, \beta=0.2, \gamma=1.5, r=2.205$ and $\Delta_{1}=0.05$, $\Delta_{2}=-0.108$. Corresponding dependences $\varphi(\psi)$ are shown in Fig. 15. One can see simultaneous existence of two equilibrium positions of the reduced system, which are stated on the straights lines $\varphi=0$ and $\varphi=\pi$. These equilibrium positions correspond to NNMs of coupled vibrations of the conservative system (17), which appear as a result of bifurcation in the vicinity of resonance.

Trajectories withdraw in time from the upper equilibrium position and approach the lower one. Thus, the upper equilibrium position corresponds to the unstable mode of coupled vibrations, and the lower one corresponds to the stable mode. Trajectories do not approach straights lines $\psi=0$ and $\psi=\pi / 2$, corresponding to the localized modes. It is necessary to develop additional investigation to determine the mode stability.

A behavior of the system (17) near resonance can be illustrated by numerical integration using the Runge-Kutta method on the interval $t \in[0 ; 500]$ for $\Omega=0.30822, c_{0}=0.4, c_{1}=0.59, c_{2}=0.01, M=10, m=0.1, \beta=0.2, \gamma=0.05, r=0.117, \varepsilon=0.1$ and $\Delta_{1}=0.05, \Delta_{2}=-0.15$ and $z_{1}(0)=0.1, z_{1}^{\prime}(0)=0, z_{2}(0)=0.01, z_{2}^{\prime}(0)=0$ for the mode of localization $\mathrm{n}$ on the coordinate $z_{1}$. Other initial values, $z_{1}(0)=0.01, z_{1}^{\prime}(0)=0, z_{2}(0)=0.1, z_{2}^{\prime}(0)=0$, are chosen for the mode of localization on the coordinate

(a)

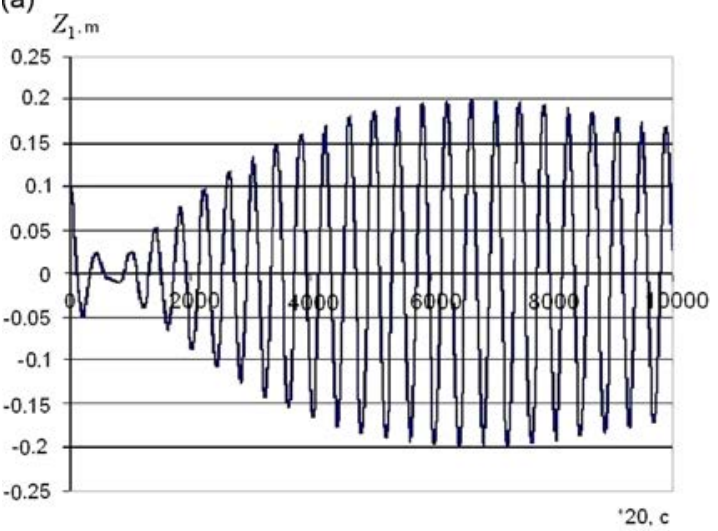

(b)

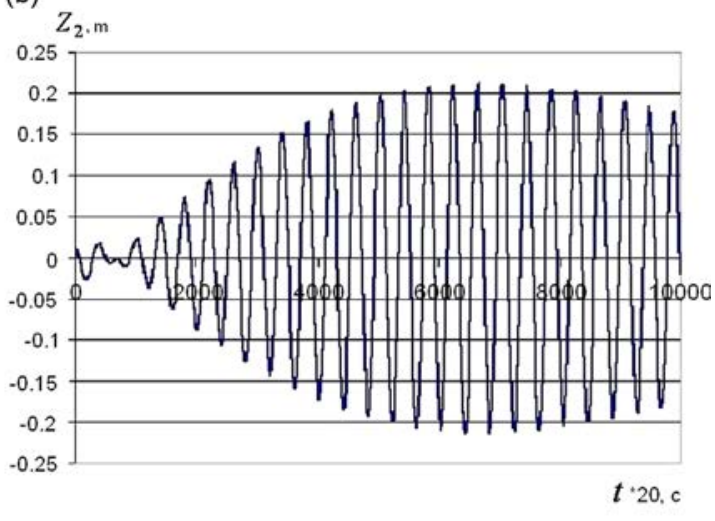

(c)

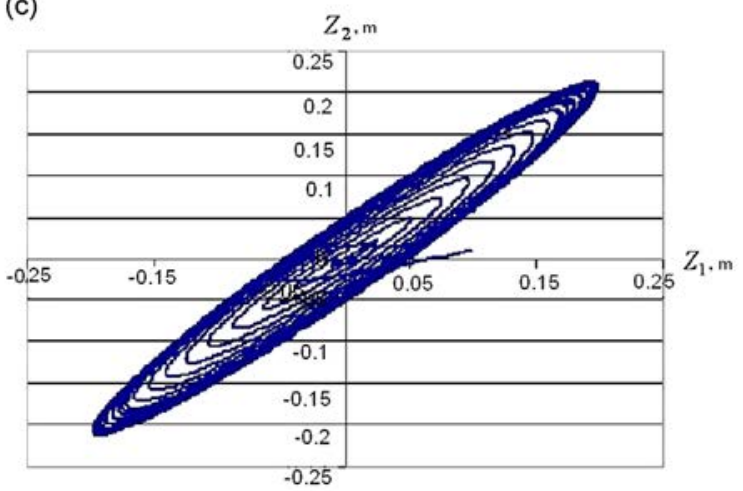

Fig. 16. Trajectories in the configuration space for the mode of localization on the coordinate $z_{1}$. 
(a)

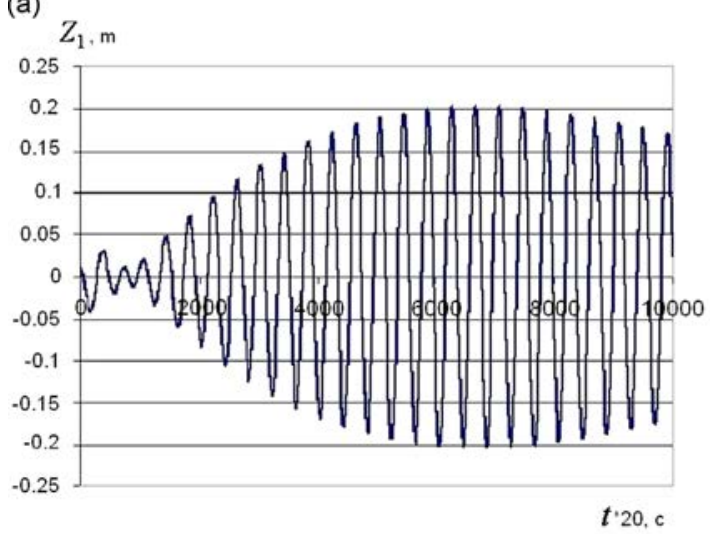

(b)

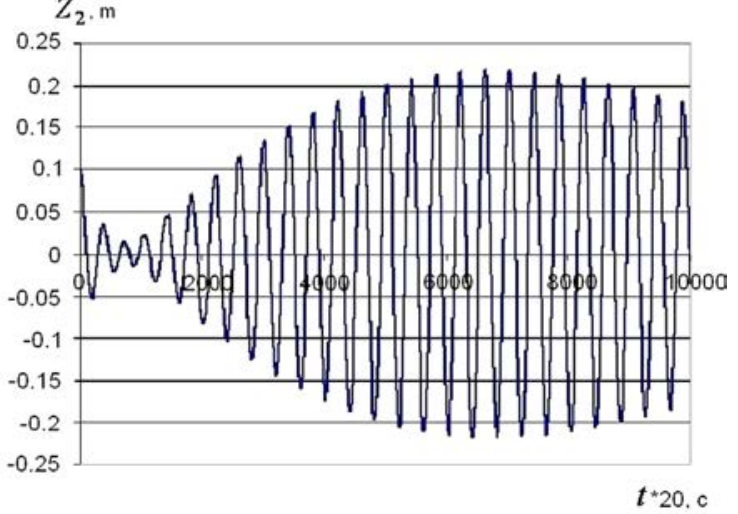

(c)

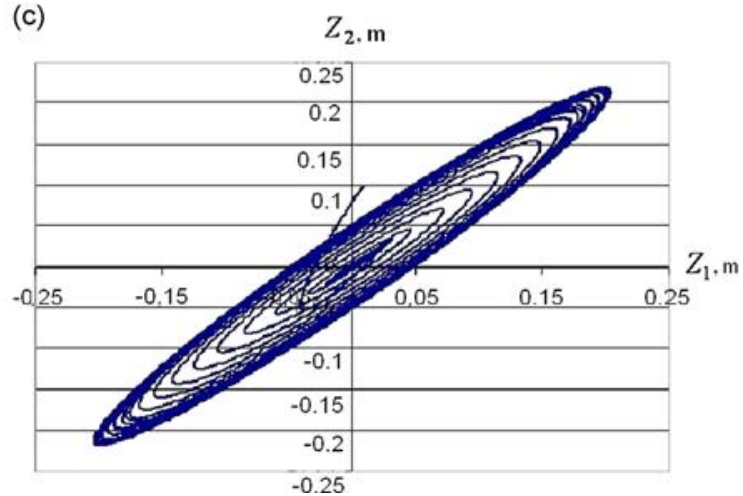

Fig. 17. Trajectories in the configuration space for the mode of localization on the coordinate $z_{2}$.

$z_{2}$. Trajectories in the configuration place are presented in Fig. 16 for the mode of localization on the coordinate $z_{1}$. Similar trajectories for the mode of localization on the coordinate $z_{2}$ are presented in Fig. 17. One can see in Figs. 16 and 17 that both localized modes are unstable, and the transfer to mode of coupled vibrations develops very quickly. Motion of the system (17) continues in the vicinity of the rectilinear nonlinear normal mode of coupled vibrations.

We can conclude that results of numerical simulation confirm reliability of main analytical results obtained for the system (13) using a procedure of passage to the reduced system.

One presents now some conclusions about a possibility of vibration localization on original coordinate $z_{1}$ (or $z_{2}$ ) of the non-autonomous system (13).

It is obtained that in the case of external resonance on the first natural frequency $\omega_{1}$, an appearance of the mode of coupled vibrations is possible when the energy reaches some value admissible for this mode bifurcation. This bifurcation exists in some time instant; and other time the system energy concentrates on the stable mode of vibrations localized on generalized coordinate $z_{1}$. This localization on generalized coordinate corresponds to the mode $x=z_{1}, y=\beta_{1} z_{1}$ of the linearized system (13). For the system parameters chosen for numerical integration in Section 3.1 (Figs. 10, 11) one has $\beta_{1}=1.4735$, which corresponds to the coupled vibration mode in original system. To guarantee a localization of the vibration energy at the nonlinear attachment, it is necessary to increase the value of $\beta_{1}$. For example, if the parameter $c_{2}$ has ten times smaller value, one has $\beta_{1}=16.5372$, and it gives the localization on the coordinate $y$, that is at the nonlinear vibration absorber.

In the case of external resonance on the second natural frequency $\omega_{2}$, an appearance of coupled vibration mode is possible, as in the preceding case, at some time instant, corresponding to some specific value of energy. This mode exists only at the time instant; in other moments of time the system vibrations are localized on the generalized coordinate $z_{2}$; namely this localized mode is stable in the vicinity of the resonance. It corresponds to the mode $x=z_{2}, y=\beta_{2} z_{2}$ of the linearized system (13). For the system parameters chosen for numerical integration in Section 3.2 (Figs. 13, 14) one has the value $\beta_{2}=-3.2235$, corresponding to coupled vibrations of the linearized system. To guarantee the vibration energy localization at the nonlinear attachment, it is necessary to increase the absolute value of $\beta_{2}$. In particular, if a mass of the absorber $m$ has ten times smaller value, one has $\beta_{2}=-45.543$; it gives a localization on the coordinate $y$, that is at the nonlinear absorber.

In the case of simultaneous existence of external and internal resonances in the system (13) the localization of energy is possible only at some time instant. At any other time the localization is not realized, and the system trajectories approach the mode of coupled motions. The following relation can describe the motion of initial system (13): $x=z_{1}+f\left(z_{1}\right)$, 
$y=\beta_{1} z_{1}+\beta_{2} f\left(z_{1}\right)$. It is clear from the relation (15) that the condition of internal resonance is $\sqrt{\left(\omega_{x}^{2}-\omega_{y}^{2}\right)^{2}+4 \omega_{y}^{2} q}=\varepsilon \delta$, i.e., it is necessary to minimize the expression $\sqrt{\left(\omega_{x}^{2}-\omega_{y}^{2}\right)^{2}+4 \omega_{y}^{2} q}$ to get into the internal resonance vicinity. The minimum can be obtained by $\omega_{x}^{2}=\omega_{y}^{2}$, and $4 \omega_{y}^{2} q \rightarrow 0$. Then one has from (16) the following equality: $\beta_{1,2}= \pm\left(\omega_{y} / \sqrt{q}\right)= \pm \sqrt{M / m}$. Thus, in the vicinity of internal resonance $y=\beta_{1}\left(z_{1}-f\left(z_{1}\right)\right)$. It is possible (theoretically) to obtain the energy localization at the nonlinear absorber using essential increasing of the parameter $\left|\beta_{1}\right|$. Taking into account the condition $\omega_{x}^{2}=\omega_{y}^{2}$ one has $c=\left(\beta_{1}^{2}-1\right) c_{2}$, i.e., there are two possibilities: either a stiffness of the elastic system $c$ is very big, or a stiffness of the absorber $c_{2}$ is very small. As a result, this case is the most unfavorable for absorption of vibrations because it is difficult to guarantee such energy localization from the physical point of view.

\section{Conclusions}

It is shown that effective analysis of the behavior of dissipative nonlinear systems in the vicinity of resonance can be carried out using the reduced system stated with respect to the system energy, the arctangent of the vibration amplitudes ratio, and the phase difference. In particular, the dynamics of the spring-mass-pendulum dissipative system (1) is investigated in the vicinity of internal resonance by means of the reduced system. It is obtained that depending on the detuning parameter and the system initial energy it can get into a region where vertical vibrations lose stability and there is a transition to motions which correspond to interaction of two NNMs of coupled vibrations. Thus, a bifurcation takes place. Eventually, if the system energy decreases, motions leave this region, the vertical vibrations become stable, and bifurcation disappears. An additional analysis of stability of the vertical vibration mode made on the basis of the equations in variations confirms that the stability depends on time: namely, a region of the vertical vibrations instability decreases with increasing of time.

Dynamics of the nonlinear dissipative system (13) with a nonlinear vibration absorber is investigated by means of the corresponding reduced systems in cases of forced resonances on the first and second natural frequencies and at simultaneous external and internal resonances. In the vicinity of the external resonance on the first natural frequency, a localization on the generalized coordinate $z_{1}$ is observed; in the vicinity of external resonance on the second natural frequency, there is a localization on the generalized coordinate $z_{2}$. Conditions of appearance of bifurcations for cases of external resonances are received: namely, an existence of the coupled vibrations depends on the energy level and it is possible only for some value of time. In the vicinity of this value of time, motions of the corresponding conservative system are close to the mode of coupled vibrations. These vibration regimes are called transient nonlinear normal vibration modes (TNNMs). In the case of simultaneous external and internal resonances the long-term localization of energy is not observed, and motions of the system come close to a mode of coupled vibrations. There is a bifurcation, namely: two modes of coupled vibrations appear, and an existence of the localized modes depends on energy level and the system parameters; it is possible for specified values of energy. In this case the localized modes are TNNMs. Conditions of localization of energy on the nonlinear vibration absorber are obtained. Favorable and unfavorable cases for the energy localization are selected. Analytical results obtained for systems under consideration are confirmed by numerical simulation.

\section{References}

[1] A.H. Nayfeh, D.T. Mook, Nonlinear Oscillations, Wiley Inerscience, New York, 1979.

[2] K.V. Avramov, Yu.V. Mikhlin, Nonlinear dynamics of elastic systems.V.1. models, methods, phenomena, Regular and Chaotic Dynamics, Moscow-Izhevsk (2010). (in Russian).

[3] A.I. Manevich, L.I. Manevitch, The Mechanics of Nonlinear Systems with Internal Resonances, Imperial College Press, London, 2005.

[4] G.S. Gorelik, A.A. Witt, Swing of an elastic pendulum as an example of two parametrically bound linear vibration systems, Journal of Technical Physics (USSR) 3 (2-3) (1933) 294-307. (in Russian).

[5] R.A. Struble, J.H. Heinbockel, Energy transfer in a beam-pendulum system, Transaction of the ASME. Journal of Applied Mechanics 29 (1963) 590-592.

[6] R.A. Struble, J.H. Heinbockel, Resonant oscillations of a beam-pendulum system, Transactions of the ASME. Journal of Applied Mechanics 30 (1963) $181-188$.

[7] F.K. Tsel'man, On pumping transfer of energy between non-linearly-coupled oscillators in third-order resonance, Applied Mathematics and Mechanics (PMM USSR) 34 (5) (1970) 957-962.

[8] C.A. Mercer, P.L. Rees, V.J. Fahy, Energy flow between two weakly coupled oscillators subject to transient excitation, Journal of Sound and Vibration 15 (3) (1971) 373-379.

[9] V.M. Starzhinskii, Applied Methods of Nonlinear Vibrations, Nauka, Moscow, 1977 (in Russian).

[10] S.A. Nayfeh, A.H. Nayfeh, Energy transfer from high to low-frequency modes in a flexible structure via modulation, ASME Journal Vibration and Acoustics 116 (1994) 203-207.

[11] O.V. Gendelman, Transition of energy to nonlinear localized mode in highly asymmetric system of nonlinear oscillators, Nonlinear Dynamics 25 (2001) $237-253$.

[12] A.F. Vakakis, O. Gendelman, Energy pumping in nonlinear mechanical oscillators: Part I. Dynamics of the underlying Hamiltonian systems, Transactions of the ASME. Journal of Applied Mechanics 68 (2001) 34-41.

[13] O. Gendelman, L.I. Manevitch, A.F. Vakakis, R. M'Closkey, Energy pumping in nonlinear mechanical oscillators: Part II. Resonance capture, Transactions of the ASME. Journal of Applied Mechanics 68 (2001) 42-48.

[14] D.M. McFarland, L.A. Bergman, A.F. Vakakis, Experimental study of non-linear energy pumping occurring at a single fast frequency, International Journal of Non-Linear Mechanics 40 (6) (2005) 891-899.

[15] A.F. Vakakis, O.V. Gendelman, L.A. Bergman, D.M. McFarland, G. Kerschen, Y.S. Lee, Nonlinear Targeted Energy Transfer in Mechanical and Structural Systems, Springer Science, 2008.

[16] A.D. Morozov, Resonances, cycles and chaos in quasi-conservative systems, Regular and Chaotic Dynamics, Moscow-Izhevsk (2005). (in Russian).

[17] H. Kauderer, Nichtlineare Mechanik, Springer-Verlag, Berlin, 1958. 
[18] R. Rosenberg, Nonlinear vibrations of systems with many degrees of freedom (in:), Advances of Applied Mechanics 9 (1966) 156-243.

[19] R.M. Rosenberg, C.P. Atkinson, On the natural modes and their stability in nonlinear two-degree-of-freedom systems, Journal of Applied Mechanics 26 (1959) 377-385.

[20] A. Vakakis, L. Manevitch, Yu. Mikhlin, V. Pilipchuk, A. Zevin, Normal Modes and Localization in Nonlinear Systems, Wiley Interscience, New York, 1996.

[21] Yu.V. Mikhlin, K.V. Avramov, Nonlinear normal modes for vibrating mechanical systems. Review of theoretical developments, Applied Mechanics Review 63 (2010) 4-20.

[22] K.V. Avramov, Yu.V. Mikhlin, Review of applications of nonlinear normal modes for vibrating mechanical systems (pages), Applied Mechanics Review 65 (2) (2013) 20, http://dx.doi.org/10.1115/1.4023533.

[23] V.N. Pilipchuk, Nonlinear Dynamics: Between Linear and Impact Limits, Springer-Verlag, Berlin, 2010.

[24] F. Wang, A. Bajaj, K. Kamiya, Nonlinear Normal Modes and Their Bifurcations for an Inertially-Coupled Nonlinear Conservative System, Nonlinear Dynamics 42 (3) (2005) 233-265.

[25] A.H. Nayfeh, Perturbation Methods, Wiley, New York, 1973.

[26] C. Hayashi, Nonlinear Oscillations in Physical Systems, McGraw-Hill, New York, 1964. 